\title{
AML-induced osteogenic differentiation in mesenchymal stromal cells supports leukemia growth
}

\author{
V. Lokesh Battula, ${ }^{1,2}$ Phuong M. Le, Jeffrey C. Sun, ${ }^{1}$ Khoa Nguyen, ${ }^{1}$ Bin Yuan, ${ }^{1}$ Ximin Zhou, ${ }^{1}$ \\ Sonali Sonnylal, ${ }^{1}$ Teresa McQueen, ${ }^{1}$ Vivian Ruvolo, ${ }^{1}$ Keith A. Michel, ${ }^{3}$ Xiaoyang Ling, ${ }^{1}$ \\ Rodrigo Jacamo, ${ }^{1}$ Elizabeth Shpall, ${ }^{4}$ Zhiqiang Wang, ${ }^{5}$ Arvind Rao, ${ }^{6}$ Cheath Al-Atrash, ${ }^{4}$ \\ Marina Konopleva, ${ }^{1}$ R. Eric Davis, ${ }^{5}$ Melvyn A. Harrington, ${ }^{7}$ Catherine W. Cahill, \\ Carlos Bueso-Ramos, ${ }^{8}$ and Michael Andreeff', ${ }^{1,4}$ \\ 'Molecular Hematology and Therapy, Department of Leukemia, ${ }^{2}$ Department of Breast Medical Oncology, ${ }^{3}$ Small Animal \\ Imaging Facility, ${ }^{4}$ Department of Stem Cell Transplantation and Cellular Therapy, ${ }^{5}$ Department of Lymphoma and \\ Myeloma, and ${ }^{6}$ Department of Bioinformatics and Computational Biology, University of Texas MD Anderson Cancer \\ Center, Houston, Texas, USA. ${ }^{7}$ Orthopedic Surgery, Baylor College of Medicine, Houston, Texas, USA. ${ }^{8}$ Department of \\ Hematopathology, University of Texas MD Anderson Cancer Center, Houston, Texas, USA.
}

Genotypic and phenotypic alterations in the bone marrow (BM) microenvironment, in particular in osteoprogenitor cells, have been shown to support leukemogenesis. However, it is unclear how leukemia cells alter the BM microenvironment to create a hospitable niche. Here, we report that acute myeloid leukemia (AML) cells, but not normal $\mathrm{CD} 34^{+}$or $\mathrm{CD}_{3} 3^{+}$cells, induce osteogenic differentiation in mesenchymal stromal cells (MSCs). In addition, AML cells inhibited adipogenic differentiation of MSCs. Mechanistic studies identified that AML-derived BMPs activate Smad1/5 signaling to induce osteogenic differentiation in MSCs. Gene expression array analysis revealed that AML cells induce connective tissue growth factor (CTCF) expression in BM-MSCs irrespective of AML type. Overexpression of CTCF in a transgenic mouse model greatly enhanced leukemia engraftment in vivo. Together, our data suggest that $A M L$ cells induce a preosteoblast-rich niche in the BM that in turn enhances AML expansion.

Authorship note: P.M. Le and J.C. Sun contributed equally to this work.

Conflict of interest: The authors have declared that no conflict of interest exists.

Submitted: August 25, 2016 Accepted: May 16, 2017 Published: June 15, 2017

\section{Reference information:} JCI Insight. 2017;2(12):e90036 https://doi.org/10.1172/jici. insight.90036.

\section{Introduction}

Acute myeloid leukemia (AML) is characterized by the accumulation of immature myeloid blasts in the bone marrow (BM) and peripheral circulation. This abnormal growth of AML cells disrupts normal hematopoiesis in the BM microenvironment (1). However, it is unclear how different the composition of the AML-BM microenvironment is compared to its normal counterpart. It is also not clear whether changes in the BM microenvironment are induced directly by AML cells or indirectly through other factors.

The BM microenvironment contains endosteal and vascular niches. The vascular niches, which consist mostly of sinusoidal endothelial cells and pericytes, have been reported to support AML growth and chemoresistance $(2,3)$. The endosteal niche comprises pre/proosteoblasts, mature osteoblasts, osteocytes, osteoclasts, and mesenchymal stromal cells (MSCs) (3). MSCs have been reported to support AML cell survival and BM engraftment and promote drug resistance (4-6). MSCs exhibit an extensive self-renewal capacity and are able to differentiate into three major mesodermal lineages: osteoblasts, adipocytes, and chondrocytes $(7,8)$. Osteogenic differentiation of MSCs results in expression of osteogenic proteins, including osterix, osteopontin, RUNX2, and tissue nonspecific alkaline phosphatase (TNAP, also known as ALP) $(9,10)$. It is not clear if and how AML cells induce changes in differentiation potential of MSCs.

BMPs are members of the transforming growth factor- $\beta$ superfamily that are able to induce osteogenic differentiation during embryonic development (11-13). BMPs exert their biological effects by signaling through two type I serine/threonine kinase receptors, RIA and RIB, which are transduced by Smad1, -4 , and -5 transcription factors. Previous reports suggest that elevated BMP-mediated signaling in AML patients promotes expression of antiapoptotic genes (14) and promotes AML cell growth (15). However, it is not clear how elevated BMP signaling affects the surrounding stroma in the AML-BM. BMPs have been 
reported to induce connective tissue growth factor (CTGF) expression in MSCs (16). CTGF is a member of the CCN family of proteins and has been shown to regulate MSC differentiation (17). CTGF is highly expressed and prognostic in acute lymphoid leukemias (18). Inhibition of CTGF activity by anti-CTGF monoclonal antibody increased the survival of leukemia-bearing mice (19).

Here, we hypothesized that AML cells mediate functional alterations in stromal cells to induce preosteoblastic-rich niches in the BM microenvironment. To address this, we examined AML-MSCs and discovered that they are induced to undergo osteogenic differentiation. Human AML xenograft and syngenic mouse AML models were used to study AML-stroma interactions in vivo and confirm that AML cells alter the differentiation potential of MSCs by inducing CTGF to create specialized hospitable niches that support their growth. Investigations of primary patient-derived BM biopsies confirmed these findings.

\section{Results}

AML-MSCs are phenotypically distinct from normal MSCs. We isolated MSCs from the BM of AML patients (AML-MSCs; $n=24$ ) aged 40-70 years and age-matched normal healthy donors (N-MSCs; $n=11$ ). Patient characteristics, including AML subtype, are summarized in Supplemental Table 1 (supplemental material available online with this article; https://doi.org/10.1172/jci.insight.90036DS1). Morphologically, AML-MSCs are polygonal or irregularly shaped and are much larger than spindle-shaped N-MSCs (diameter, 100-150 $\mu \mathrm{M}$ versus 40-60 $\mu \mathrm{M} ; P<0.01$ ) (Supplemental Figure 1A). Growth analysis of AMLMSCs and N-MSCs showed that AML cells grow 2- to 3-fold more slowly $(P<0.01)$ than N-MSCs (Supplemental Figure 1B). Furthermore, BrdU pulse and propidium iodide (PI) labeling assay revealed that $9.6 \% \pm 4.1 \%$ of $\mathrm{N}-\mathrm{MSC}$ in S-phase were positive for BrdU uptake, versus only $2.59 \% \pm 0.38 \%$ of AML-MSCs $(P<0.001$; Supplemental Figure 2), indicating a slower proliferation rate for AML-MSCs. The cell surface phenotypes of AML-MSCs and N-MSCs revealed that BM-MSC-associated markers, including CD44, CD51, CD73, CD90, CD105, CD106, CD140b, CD146, and SUSD2, were expressed on both cell types at equal intensities (Supplemental Figure 3). Neither CD45 nor CD31 was expressed on either AML-MSCs or N-MSCs (Supplemental Figure 3).

Flow cytometry revealed that TNAP (clone W8B2), known to be expressed on osteoprogenitor cells (20), mature osteoblasts, and naive MSCs (21), was significantly upregulated in AML-MSCs compared with N-MSCs (Figure 1A). In the cohort of primary MSC samples isolated from AML patients with different disease status (newly diagnosed or in remission or relapsed; $n=29$ ), the average mean fluorescence intensity (MFI) of TNAP was approximately 10 -fold higher than that in N-MSCs $(n=11$; Figure 1B, $P<0.01)$. The median MFI for N-MSCs was 146 , versus 1,033 for AML-MSCs. Only $\leq 10 \%$ of AML-MSCs showed TNAP MFI values <500, suggesting that most AML subtypes overexpress TNAP (Supplemental Table 1). However, MFI of other cell surface markers analyzed was not significantly changed between AML- and N-MSCs types (Supplemental Figure 3 and 4).

$A M L-M S C$ s are primed for osteogenic differentiation. Because the osteogenic differentiation marker TNAP was upregulated in AML-MSCs compared with N-MSCs, we determined whether other osteogenic lineage-associated genes were also upregulated in AML-MSCs. mRNA expression of several genes associated with osteogenic differentiation determined by qRT-PCR was upregulated by 3- to 10-fold in AML-MSCs compared with N-MSCs (Figure 1C, $n=3$ ), including transcription factors RUNX2 and osterix and the cell surface or extracellular matrix-associated genes osteopontin and TNAP. Interestingly, AML-MSCs but not N-MSCs stained positive for ALP enzyme activity by the BCIP/NBT substrate assay (days 0, Figure 1D). However, AML-MSCs on day 0 (before induced osteogenic differentiation) were not positive for Alizarin Red S (which stains mineral depositions that are usually observed in mature osteoblasts), suggesting that these cells are osteoprogenitor cells but not mature osteoblasts (Figure 1, D and E). When exposed to osteogenic differentiation medium, AML-MSCs differentiated into mature osteoblasts (ALP and Alizarin Red S positive) in 1-2 weeks, whereas N-MSCs took approximately 3 weeks (Figure 1, D and E). These data suggest that AML-MSCs are primed to differentiate into the osteogenic lineage.

To determine whether the induction of osteogenic differentiation in MSCs is primed by leukemia cells in the BM, we cocultured N-MSCs with OCI-AML3 or cord blood-derived (CB-derived) CD $34^{+}$or CD $33^{+}$ cells for 3 or 5 days and measured TNAP expression by flow cytometry. Cells were gated on the CD45CD $90^{+}$population to examine TNAP expression specifically in MSCs (Supplemental Figure 5). Interestingly, we found that TNAP expression was upregulated by more than 10- to 15-fold in N-MSCs cocultured with OCI-AML3 cells compared with $\mathrm{CB}$-derived $\mathrm{CD} 34^{+}$or $\mathrm{CB}$-derived $\mathrm{CD} 33^{+}$cells or cells cultured alone 
A

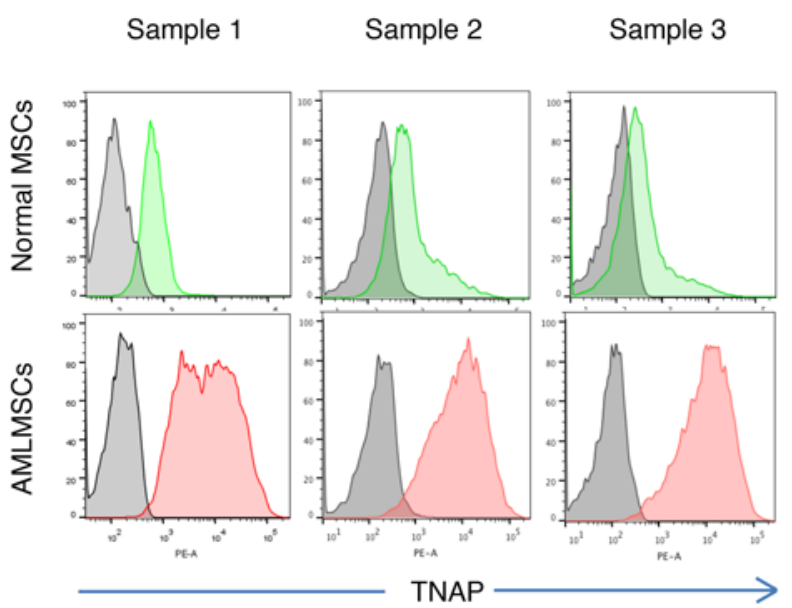

C

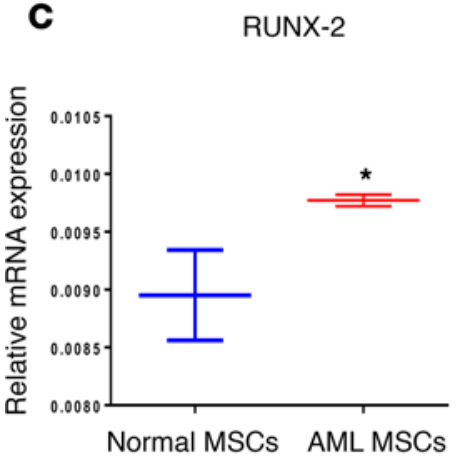

D

Before differentiation

After differentiation

Day 0

Day 7

Day 14

Day 21
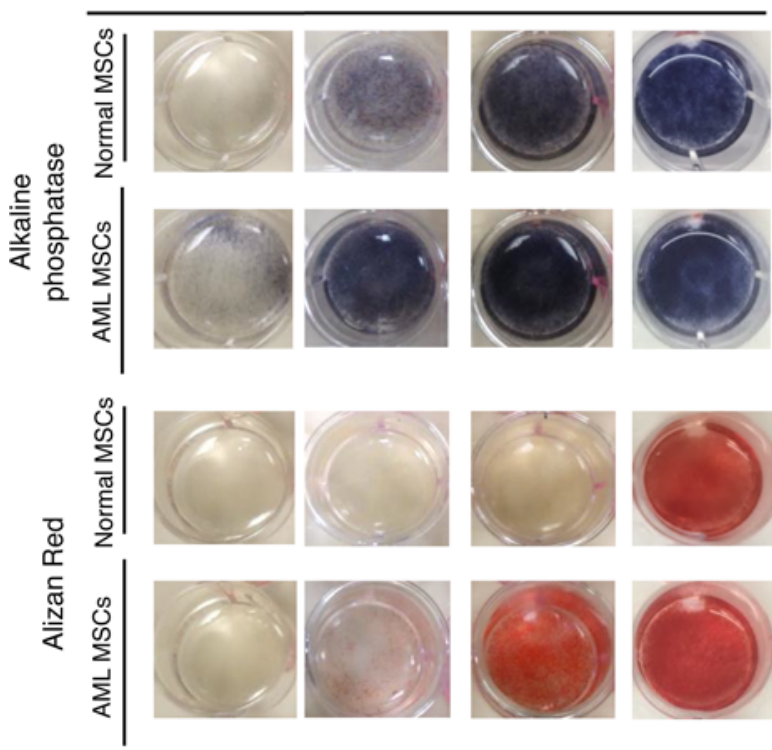

B

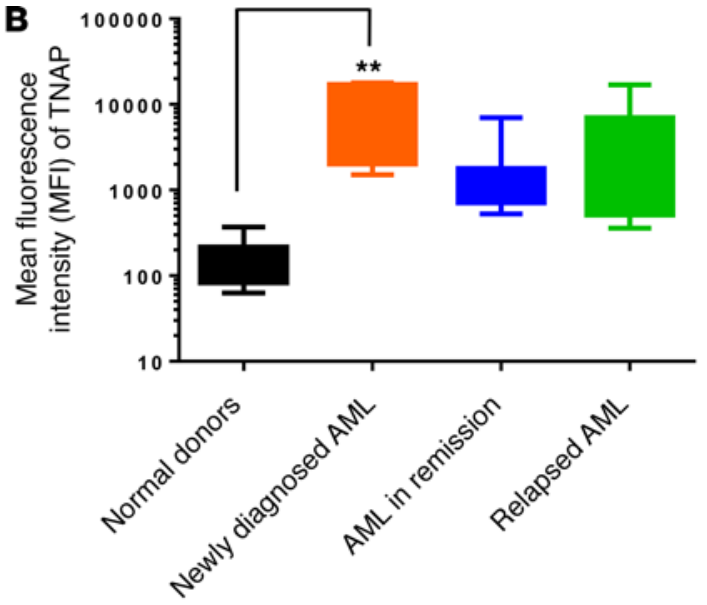

Osteopontin

TNAP
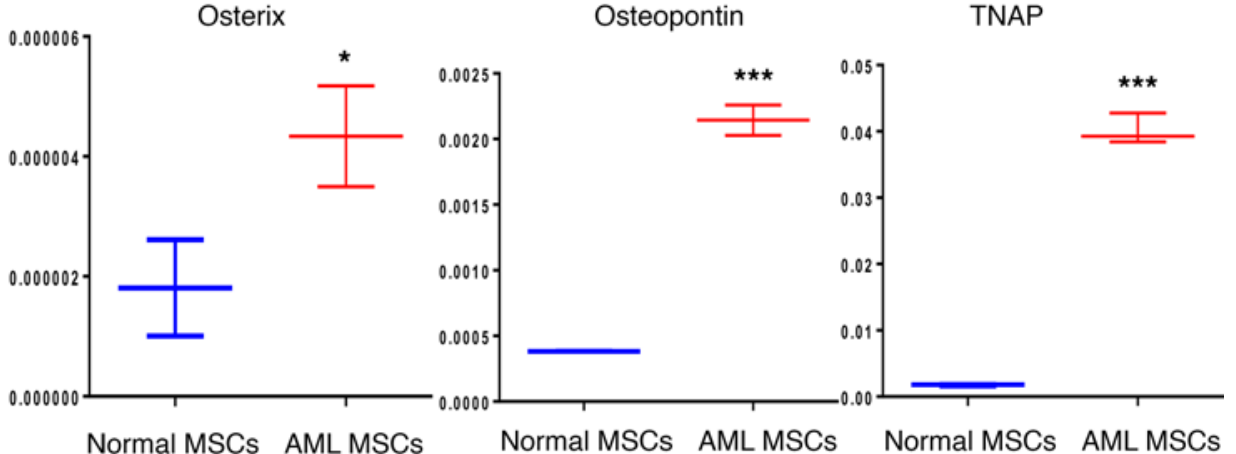

E
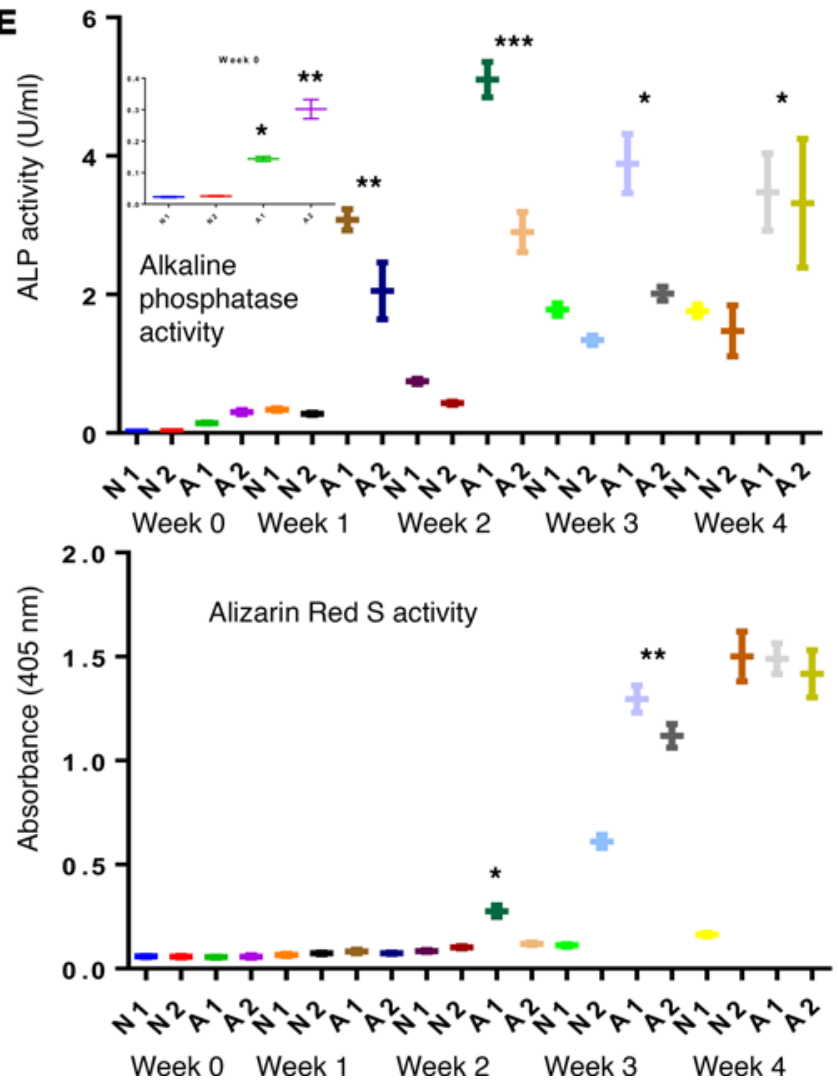
Figure 1. Acute myeloid bone marrow-derived mesenchymal stromal cells are primed to differentiate into osteoblasts. (A) Tissue nonspecific alkaline phosphatase (TNAP) expression was analyzed by flow cytometry on normal donor-derived (Normal-MSCs) (green) or acute myeloid bone marrow-derived mesenchymal stromal cells (AML-MSCs) (red) over unstained cells (gray). Cells were incubated with anti-TNAP antibody (clone W8B2) conjugated with phycoerythrin (PE). The TNAP-stained cells were overlaid on unstained cells; representative histograms ( $n=3$ for each cell type) are shown. Data were analyzed by Flowjo software. (B) MFI of normal MSCs (N-MSCs) $(n=11)$ or AML-MSCs $(n=29)$ stained with TNAP antibody were determined. AML samples with different disease status, including newly diagnosed $(n=6)$ or remission $(n=8)$ or relapsed $(n=15)$, were graphed separately. (C) mRNA expression of osteoprogenitor-associated genes, RUNX2, osterix, osteopontin, and TNAP, in N- and AML-MSCs was measured by qRT-PCR. GAPDH served as an equal loading control. (D) Alkaline phosphatase activity was determined by an enzyme assay in N- and AML-MSCs ( $n=3$ for each) cultured in the presence or absence of osteogenic differentiation medium for 3 weeks. At the end of each week (days 7, 14, and 21), the cells were incubated with FAST BCIP/NBT substrate or Alizarin Red S stain and images acquired. (E) Alkaline phosphatase enzyme activity and absorbance at $405 \mathrm{~nm}$ for Alizarin Red S staining were quantitated as described in the methods section. Statistical data were analyzed by GraphPad Prism software. One-way ANOVA was used for comparison of 3 or more groups and unpaired Student's $t$ test was used for comparisons of 2 groups. $\left({ }^{*} P<0.05,{ }^{* *} P<0.01,{ }^{* * *} P<0.001\right.$ versus control). Dunnett's multiple comparison test was used to check the statistical significance in difference between multiple groups.

(Figure 2, A and B and Supplemental Figure 6). In addition, N-MSCs pretreated with OCI-AML3 cellderived conditioned medium (OCI-AML3-CM) for 5 days differentiated twice as quickly as those treated with control medium (Figure 2C). OCI-AML3-CM-pretreated MSCs exhibited twice the ALP activity and Alizarin Red S staining as MSCs pretreated with control medium in a time-dependent manner (Figure 2D). In addition, osterix, RUNX2, osteopontin, and TNAP were upregulated by 3- to 10-fold in N-MSCs cocultured with OCI-AML3 compared with N-MSCs cultured alone (Figure 2E), suggesting that AML cells induce osteogenic differentiation in MSCs. To validate these findings further, N-MSCs were cocultured with 2 additional AML cell lines including Molm13 and HL60 for 5 days. MSCs were isolated by FACS as explained above using cell surface phenotype $\mathrm{CD} 90^{+} \mathrm{CD} 45^{-}$, and mRNA expression of osteogenic genes, including osterix, RUNX2, osteopontin, TNAP, Col1A1, and bone sialoprotein, was analyzed using qRTPCR. We found that most of the osteogenic markers were upregulated 2- to 10-fold in MSCs cocultured with AML cells compared with MSCs cultured alone (Supplemental Figure 7, A and B), suggesting that AML cells induce osteogenic differentiation in MSCs.

AML-MSCs are unable to differentiate into adipocytes. We previously reported that AML-MSCs lack the capacity to differentiate into functional adipocytes (22). To extend these observations, we cultured AMLMSCs and N-MSCs in adipogenic differentiation induction medium; RNA was extracted from the cells on days $0,7,14$, and 21 during differentiation, and expression of $a P 2, P P A R \gamma$, and lipoprotein lipase was determined by qRT-PCR. As expected, expression of these adipogenic differentiation genes in N-MSCs increased steadily over time during differentiation. However, expression of these markers was not induced in AML-MSCs under similar conditions (Figure 3A). Furthermore, less than 3\% of AML-MSCs cultured under our adipogenic differentiation conditions produced Oil Red O-positive adipocytes compared with approximately $80 \%-90 \%$ of the N-MSCs. In fact, the total number of adipocytes generated from N-MSCs was 20- to 30-times higher than that derived from AML-MSCs (Figure 3, B and C). Moreover, N-MSCs pretreated with AML-CM for 5 days and then subjected to adipogenic differentiation for 7 days showed 2 to 4-fold lower expression of adipogenic markers, including aP2, PPAR $\gamma$, and lipoprotein, than observed in the N-MSCs treated with control medium (Figure 3D). These data indicated that AML-MSCs were unable to differentiate into adipocytes and that AML cells contributed to this differentiation block.

Next, AML-MSCs and N-MSCs were tested for their capacity to differentiate into chondrocytes. Culturing both cell types in chondrogenic differentiation medium resulted in chondrocytic nodules. However, the AML-MSC-derived nodules were much smaller and more irregularly shaped than the sphere-shaped chondrocytic nodules derived from N-MSCs. In addition, both N-MSC- and AML-MSC-derived nodules stained positive for Alcian Blue and collagen type-1 (Supplemental Figure 8, A and B). These data suggested that AML-MSCs have lower chondrogenic differentiation potential than N-MSCs.

AML-induced osteogenic differentiation of MSCS was validated in a mouse model of human BM. We developed a human bone implant (HBMI) mouse model of human BM by subcutaneous implantation of human bone fragments freshly collected from patients undergoing hip replacement surgery into the flanks of NSG mice (Supplemental Figure 9A) to validate AML-induced osteogenic differentiation in vivo. CT scans using a gold nanoparticle-derived contrast agent revealed that, by 4 weeks after implantation, the bone implants received their blood supply from the host vasculature (Figure 4A and Supplemental Figure 9B). Strong staining with OsteoSense 750EX dye (which binds to hydroxyapatite) injected intravenously into the HBMI mice demonstrated active bone restoration (Supplemental Figure 9C). To investigate leukemia engraftment, we transplanted Molm13 cells expressing GFP and firefly luciferase intravenously into HBMI mice 4 weeks after 
A
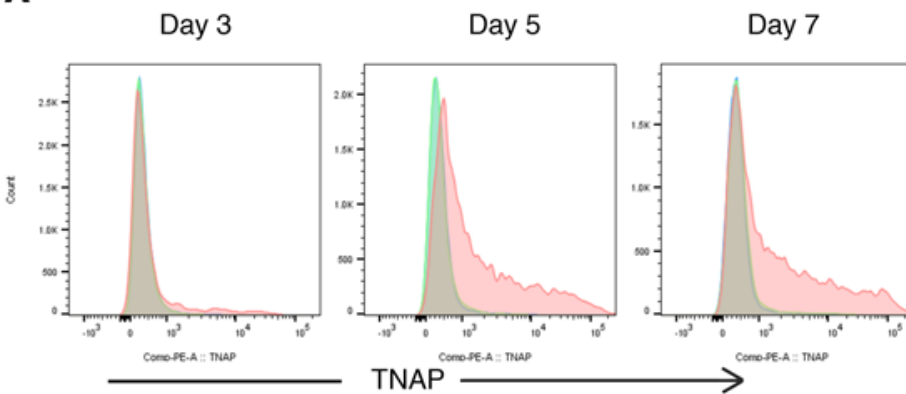

Normal-MSCs alone

Normal-MSCs + Cord blood-CD34 ${ }^{+}$cells

Normal-MSCs + OCI-AML3 cells
B

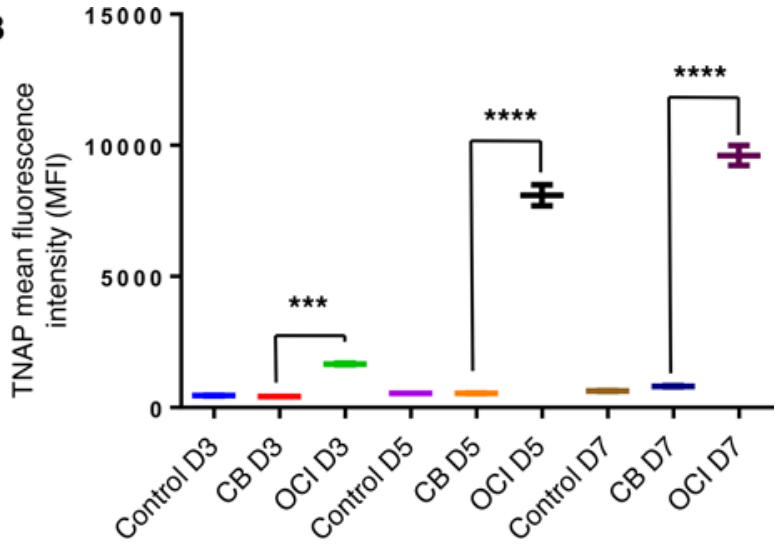

C

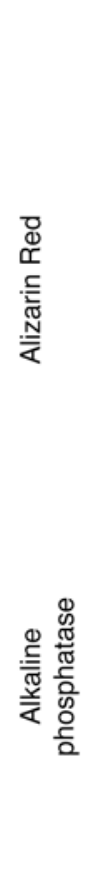

Week 1 differentiation
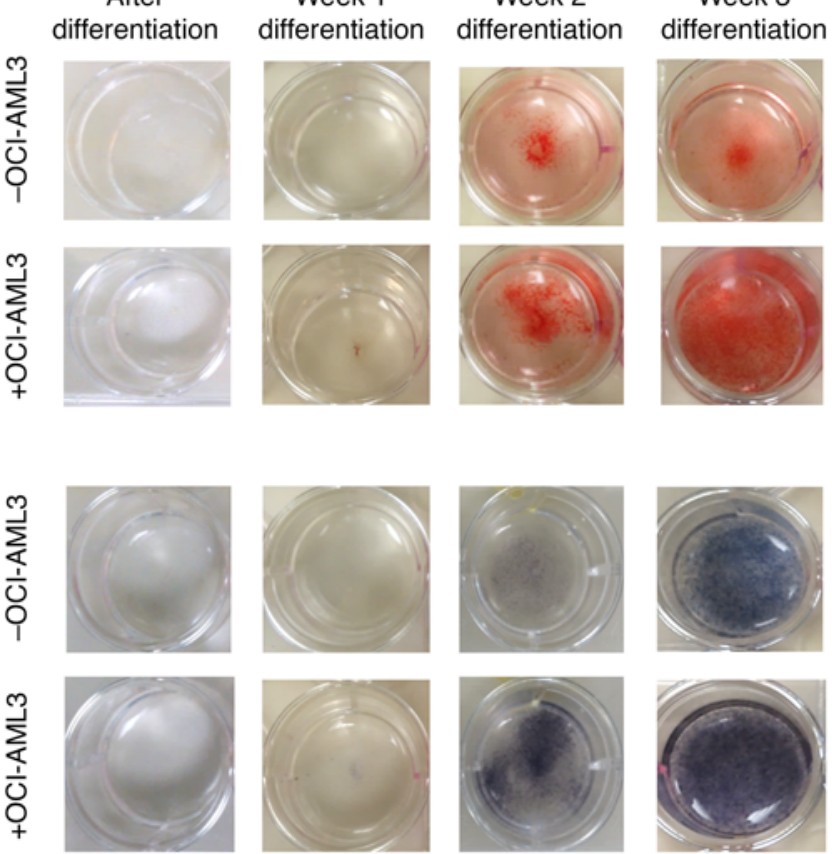

D
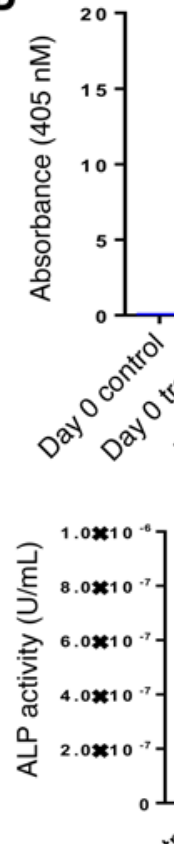

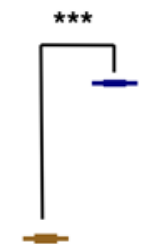

\section{E}

RUNX2

Osterix

Osteopontin

Alizarin Red S

activity

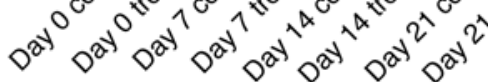

Alkaline

phosphatase

activity
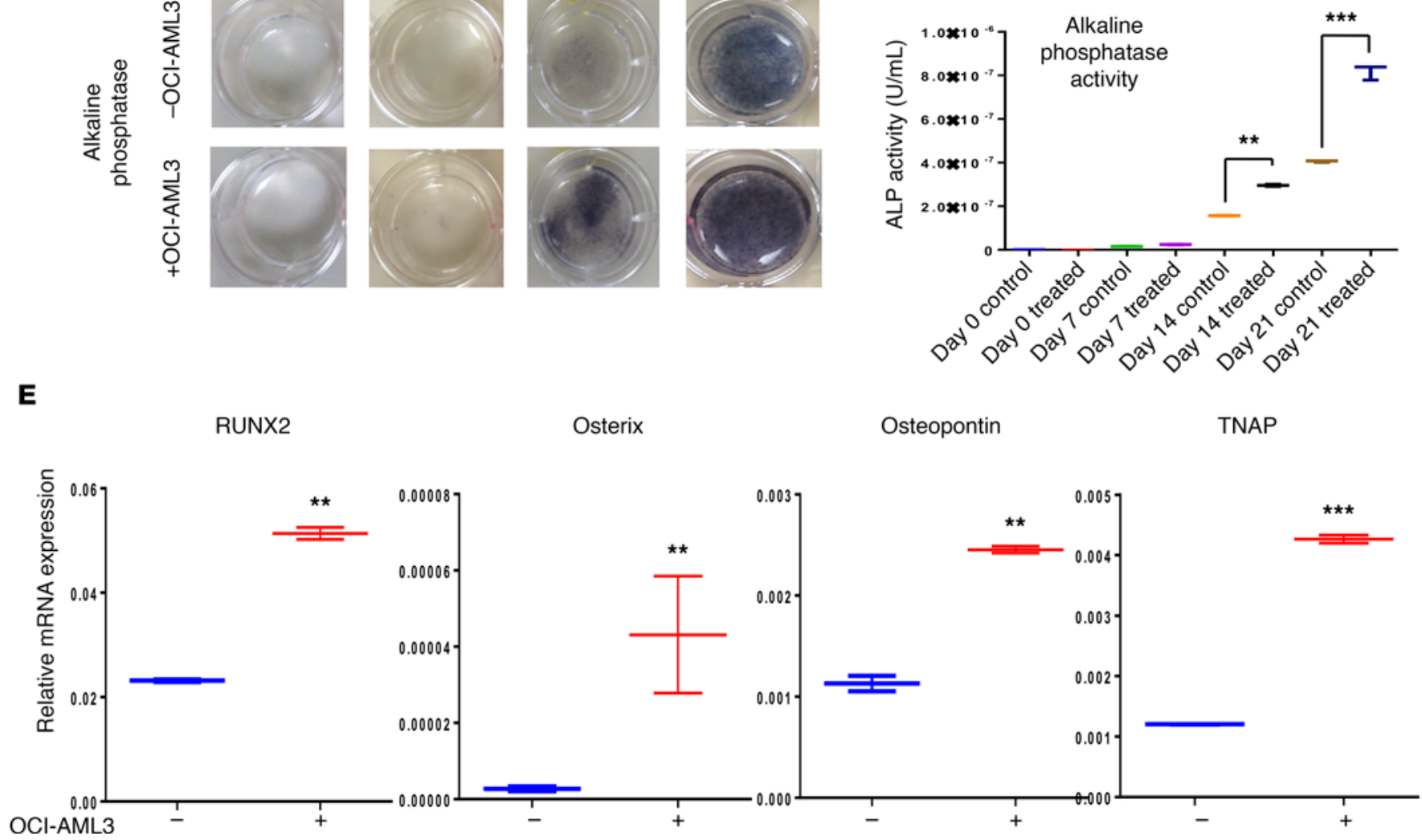

Figure 2. AML cells induce osteogenic differentiation in N-MSCs. (A) N-MSCs were cocultured with OCI-AML3 cells (red) or with cord blood-derived CD34+ cells (green) or N-MSCs cultured alone (blue) for 3, 5, or 7 days, and TNAP expression was analyzed by LSR-Il flow cytometer $(n=3)$. Data were analyzed and histograms were generated by Flowjo software. (B) MFI of TNAP expression was quantified in N-MSCs cocultured with cord blood-derived CD34 ${ }^{+}$cells or OCI-AML3 for 3 or 5 days. (C and D) N-MSCs were cultured with or without OCI-AML3 cell-derived conditioned medium (OCI-AML3-CM) for 5 days before 
long-term (3 weeks) culture in osteogenic differentiation medium. N-MSCs were subjected to Alizarin Red S staining or ALP staining on days 0 (predifferentiation), 7 (week 1), 14 (week 2), and 21 (week 3) of differentiation. (E) mRNAs from N-MSCs cultured with or without OCI-AML3-conditioned medium were examined for expression of indicated osteolineage-associated genes by qRT-PCR $(n=3)$. GAPDH served as an equal loading control. Two-way ANOVA was used for comparisons of 3 or more groups and unpaired Student's $t$ test was used for comparisons of 2 groups $\left({ }^{* *} P<0.01,{ }^{* * *} P<0.001\right.$, ${ }^{* * * *} P<$ 0.0001 versus control). In addition, Tukey's multiple comparison test was also performed for the data in $\mathbf{B}$ and $\mathbf{D}$.

bone fragment implantation. As expected, the leukemia cells strongly engrafted within the regions of HBMI implantation, as revealed by in vivo bioluminescence imaging (Supplemental Figure 9D) and H\&E staining (Supplemental Figure 9E) as early as 10 days after transplantation. Phenotypic characterization of HBMI-derived MSCs revealed that these cells expressed MSC-associated proteins, including CD44, CD90, CD140b, CD73, CD105, CD106, CD166, CD51, and SUSD2, and were negative for CD45 and CD31 (Supplemental Figure 10). When cultured in osteogenic or adipogenic differentiation medium, these MSCs developed into Alizarin Red S-positive osteoblasts and Oil Red O-positive adipocytes (Supplemental Figure 11), suggesting that components of the BM microenvironment in HBMI mice are intact and functional. These data indicate that the HBMI model is useful for studies related to factors regulating leukemia-stroma interactions.

To determine whether leukemia cells induce osteogenic differentiation in BM stromal cells in vivo, we injected Molm13 AML cells intravenously into the HBMI mouse model. After HBMI implantation, one group of mice received PBS solution (PBS; $n=10)$ and another group received Molm13 cells $(1 \times$ $10^{6}$ cells/mouse; $n=10$ ) in $100 \mu \mathrm{PBS}$. After 4 weeks, the mice were sacrificed and the bone fragments from each mouse were examined by immunohistochemistry (IHC) with the Opal multiplex tissue staining approach. Sections were costained with osteogenic markers, including osterix and RUNX2, and the hematopoietic marker CD45. All antibodies were human specific and did not recognize mouse antigens. We found that, in bone fragments derived from control mice, RUNX2 and osterix expression was restricted to the endosteal region of the bone (Figure 4B). However, in bone fragments from the Molm13 group, RUNX2 and osterix expression was upregulated in both the endosteal surface and medullar cavity, indicating greater osteogenic activity in HBMI mice harboring leukemia cells (Figure 4B). We also assessed the absolute number of osterix ${ }^{+} \mathrm{CD} 45^{-}$or RUNX2 ${ }^{+} \mathrm{CD} 45^{-}$stromal cells in bone fragments from Molm 13 and control groups using inForm image analysis software and determined that both osteogenic stromal types were present at a 5- to 7-fold higher rate in bone fragments from mice treated with Molm13 cells as compared with controls (Figure $4 \mathrm{C}$ ), indicating that the increased osteogenic activity in leukemic BM is due to osteogenic differentiation of stromal cells.

$A M L-B M$ displays higher osterix and RUNX2 expression than normal BM. Next, we examined AML patient $\mathrm{BM}$ biopsies and normal BM specimens ( $n=5$ each) for osteogenic expression by IHC staining. In the AML-BM biopsy specimens, osterix and RUNX2 were expressed not only in the bone-lining cells, but also in the BM cavity, matching the HBMI findings. In contrast, these proteins were not expressed in the marrow regions of the normal BM (Figure 4, D and E). Overall, osterix and RUNX2 were upregulated 3 and 6-fold, respectively, in AML-BM specimens compared with normal BM specimens $(P<0.01)$, further suggesting that AML-BM specimens exhibit increased osteogenic activity.

AML cells induce osteogenic differentiation in MSCs through BMP-mediated signaling. To investigate the mechanisms involved in AML-induced osteogenic differentiation in MSCs, we focused on the role of BMP-mediated signaling, as the induction of osteogenic differentiation by BMPs is well established (23). N-MSCs were cultured in the presence or absence of OCI-AML3-CM, and the activation of Smad1/5 proteins was determined by Western blotting. Interestingly, the Smad1/5 proteins were phosphorylated in N-MSCs cultured with OCI-AML3-CM in a time-dependent manner, with the signal peaking at 60 minutes after treatment (Figure 5A). To determine whether Smad1/5 phosphorylation occurs though BMP ligands, but not through cross-talk with other signaling pathways, we cultured N-MSCs with OCI-AML3-CM for 60 minutes in the presence or absence of the BMP type-1 receptor-specific inhibitor LDN-212854 (24). Interestingly, we observed a progressive decrease in OCI-AML3-CM-induced Smad1/5 phosphorylation in MSCs with increasing concentrations of LDN-212854 (10-1,000 ng/ml), suggesting that Smad1/5 activation in MSCs is mediated though a BMP ligand secreted by AML cells (Figure 5B).

To determine the role of BMP signaling in AML-induced osteogenic differentiation in MSCs, N-MSCs were cultured in the presence or absence of OCI-AML3-CM for 5 days with or without LDN-212854 and analyzed for osteogenic markers by qRT-PCR. Expression of osterix, RUNX2, and osteopontin was 2- to 3-fold higher in N-MSCs cultured in OCI-AML3-CM (Figure 5C) compared with the control medium. In 
A

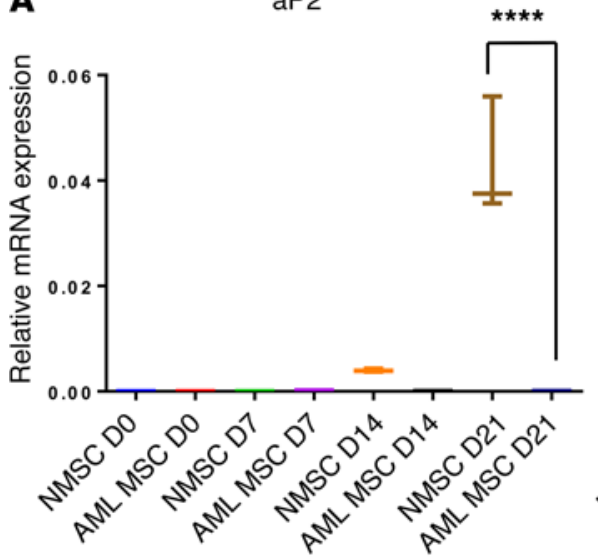

B

Sample 1

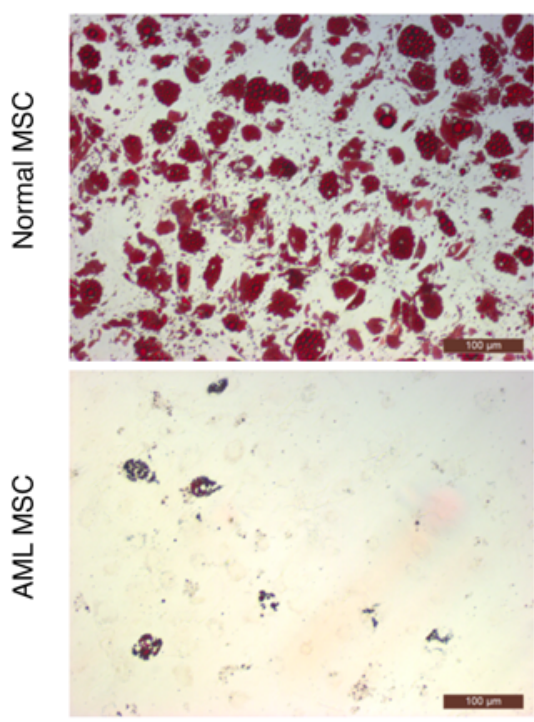

D aP2

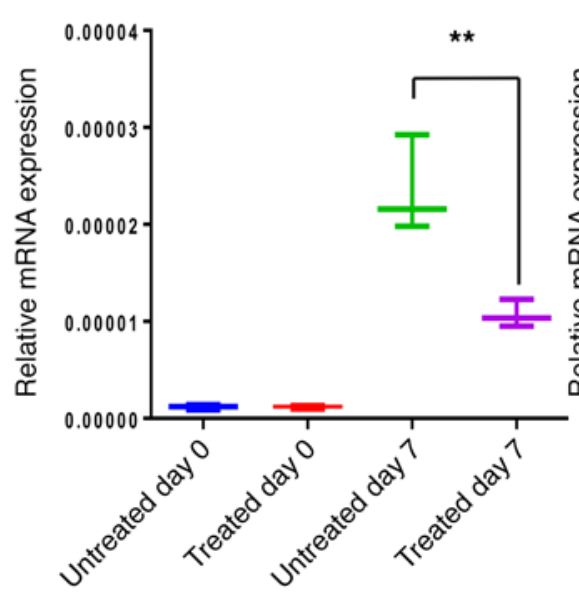

Lipoprotein lipase

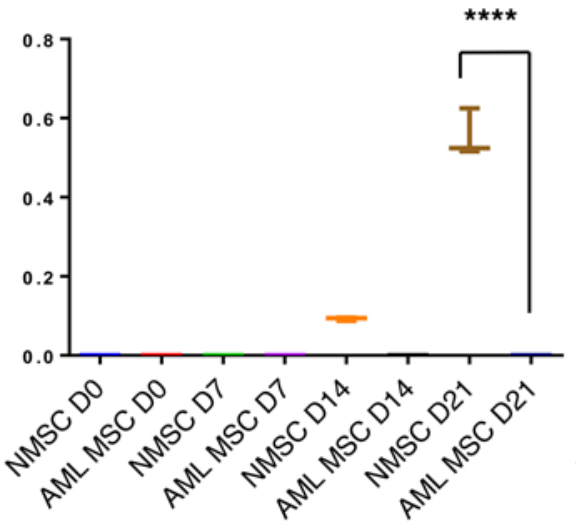

Sample 2

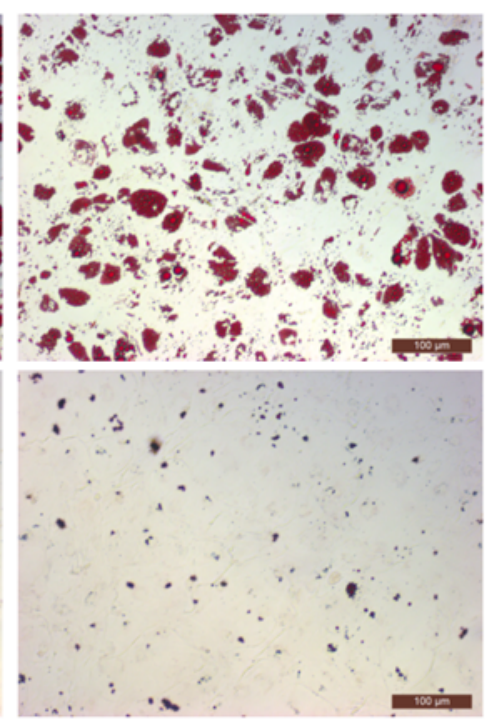

Lipoprotein lipase

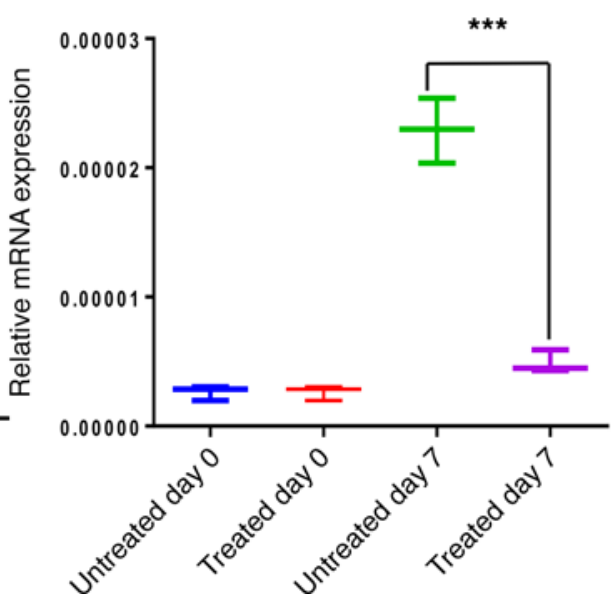

C
PPAR gamma
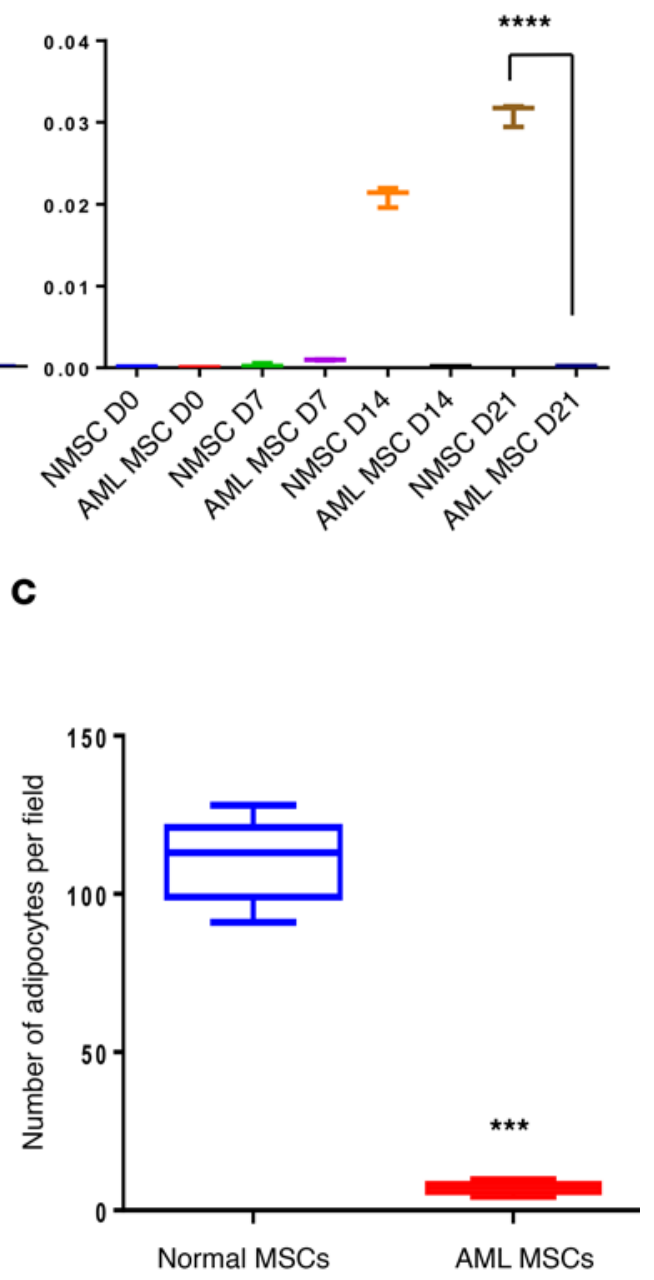

PPAR gamma

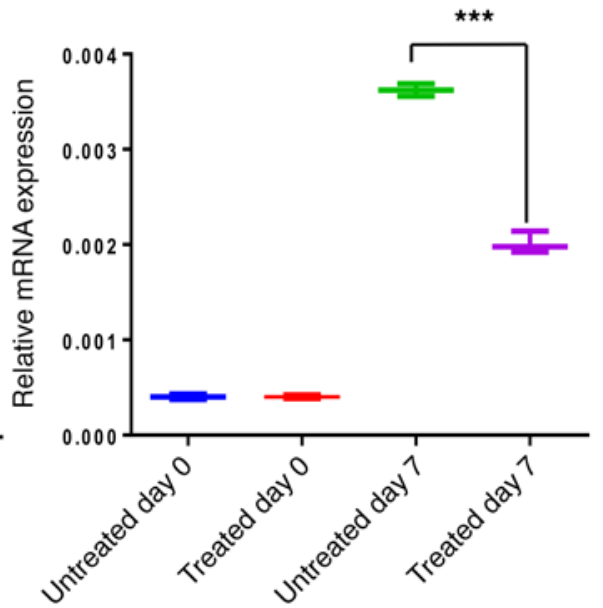

Figure 3. AML cells inhibit adipogenic differentiation in MSCs. (A) N- and AML-MSCs were cultured in adipogenic differentiation medium for 3 weeks. mRNA expression of adipocyte-associated genes, $a P 2$, lipoprotein lipase, and PPAR $\gamma$, in N- and AML-MSCs before and after induction of differentiation was analyzed weekly by qRT-PCR. (B) N- or AML-MSCs ( $n=5$ each group) were cultured in adipogenic differentiation medium for 28 days. On day 28, the adipocytes were stained with Oil Red 0 . Scale bar: $100 \mu \mathrm{m}$. (C) Oil Red 0-positive cells (i.e., adipocytes) were counted in 10 microscopic fields per sample. (D) N-MSCs were pretreated with OCI-AML3-conditioned medium for 5 days before they were cultured in adipogenic differentiation 
medium for 7 days. Expression of indicated adipogenic lineage-associated genes before induction of differentiation and on day 7 was analyzed by qRT-PCR. GAPDH served as an equal loading control. Two-way ANOVA was used for comparisons of 3 or more groups and unpaired Student's $t$ test was used for comparisons of 2 groups $\left({ }^{* *} P<0.01,{ }^{* *} P<0.001 .{ }^{* * *} P<0.0001\right.$ versus control). In addition, Tukey's multiple comparison test was also performed for multiple data sets.

the presence of LDN-212854, however, osteogenic marker expression was inhibited by more than 10-fold (Figure 5C). Next, to determine BMP expression in AML cells, we analyzed mRNA expression of BMP1, BMP2, BMP4, BMP6, BMP7, and BMP10 in AML cell lines, including Thp1, HL60, Molm13, OCIAML3, and Molm14, by qRT-PCR. We found that OCI-AML3 cells express BMP1, BMP4, BMP7, and BMP10; Thp1 cells express BMP4; HL60 cells express BMP6 and BMP10; Molm13 cells express BMP2, BMP6, and BMP7; and Molm14 cells express BMP1, BMP4, and BMP7 (Supplemental Figure 12). These data indicate that AML cell-induced osteogenic differentiation in MSCs is mediated by BMP signaling.

Gene expression profiling reveals upregulation of CTGF in MSCS by AML in vivo. To investigate AML-induced changes in MSCs, we used a previously established murine AML model (25). Murine AML cells with genetic alterations, including MLL/ENL and MLL/ENL+FLT3-ITD or AML1/ETO9a or MLL/ ENL+FLT3-ITD p53 wt (gift from Scott Lowe, Memorial Sloan Kettering Cancer Center, New York, NY, USA), were transplanted in C57/B6 mice (10 mice/group). Mice injected with PBS served as healthy controls. BM-MSCs from mice with and without leukemia were isolated by FACS using antibodies against a combination of cell surface proteins, including Ter119-, CD31-, CD45-, $\mathrm{CD} 105^{+}, \mathrm{Sca}^{+}, \mathrm{CD}^{-} 06^{+}$, and PDGF-R $\alpha^{+}$(CD140). Total RNA from sorted MSCs was used for gene expression profiling on Illumina mouse WG6 v2.0 microarrays. The accession number for gene expression profiling data is GSE97194 (https://www.ncbi.nlm.nih.gov/geo/).

Heat mapping of genes that were differentially expressed by MSCs from AML-bearing mice, as compared with MSCs from control mice (Figure 5D), showed that many were changed substantially only by exposure to one AML genotype or were even changed in different directions by different genotypes. However, a small group of genes was consistently upregulated in AML-exposed MSCs. Among those was CTGF, whose average expression in MSCs across various AML genotypes was upregulated by 12- and 33-fold, as found using 2 array probes, compared with control MSCs (Figure 5D). CTGF is known to be overexpressed in acute leukemia and has been shown to be a poor prognosis factor in acute lymphoid leukemia (18). It is one of the most highly expressed genes during osteogenic differentiation $(26,27)$, and its expression has been shown to be induced by BMP signaling in MSCs (24). To validate whether AML cells induce CTGF expression in MSCs, we cultured N-MSCs $(n=3)$ in the presence or absence of OCI-AML3 cells (Figure 5E) or OCI-AML3-CM (Figure 5F) for 5 days. Quantitative RT-PCR revealed that CTGF expression was induced 2- to 4-fold in MSCs cultured with OCI-AML3 cells or OCI-AML3-CM compared with MSCs cultured alone (Figure 5, E and F). In addition, inhibition of BMP signaling by LDN-212854 inhibited AML-induced CTGF expression in MSCs (Figure $5 \mathrm{~F}$ ), suggesting that AML-induced BMP-mediated signaling regulates CTGF expression in MSCs.

CTGF overexpression enhances leukemia engraftment in mice. We used Colla2-CTGF-transgenic mice, in which CTGF is expressed in Col1a2-positive cells, to investigate the significance of AML-induced CTGF expression in MSCs, and we used a syngeneic mouse AML model in which mouse hematopoietic stem cells were transduced to introduce the MLL-ENL fusion gene (25). To determine whether CTGF overexpression enhanced leukemia engraftment in mice, we injected $M L L-E N L$ fusion gene-transduced AML cells intravenously into WT $(n=3)$ or Col1a2-CTGF-transgenic mice $(n=3)$. In vivo bioluminescence imaging revealed time-dependent enhancement (4-fold) of leukemia engraftment in CTGF-overexpressing mice compared with WT mice $(P<0.01$; Figure 6 , A and B). Flow cytometric analysis indicated a higher percentage of leukemia cells in the peripheral blood of CTGF-transgenic mice $(25 \% \pm 3 \%)$ than in that of WT mice ( $3 \% \pm 1.5 \%$; Figure $6 \mathrm{C})$. Immunofluorescence analysis of mouse spleens using anti-GFP antibody also revealed greater $\mathrm{GFP}^{+}$leukemia engraftment in spleens from CTGF-transgenic mice $(35 \% \pm$ $8.5 \%)$ than in those from WT mice $(4.5 \% \pm 2.5 \% ; P<0.01$; Figure $6, \mathrm{D}$ and $\mathrm{E})$.

\section{Discussion}

In this report, we demonstrated that AML-MSCs are phenotypically and functionally different from normal donor-derived MSCs. We found that osteogenic lineage-associated genes were upregulated in AMLMSCs compared with N-MSCs. However, AML-MSCs were unable to differentiate into adipocytes. Coculture of AML cells with N-MSCs upregulated osteogenic marker expression in MSCs, suggesting that AML 
A

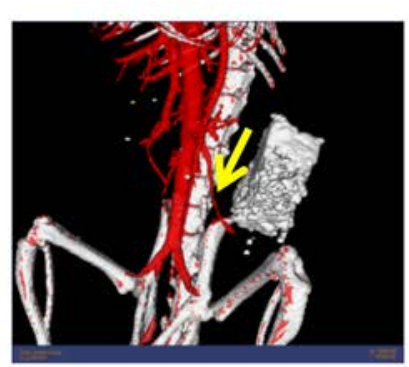

B

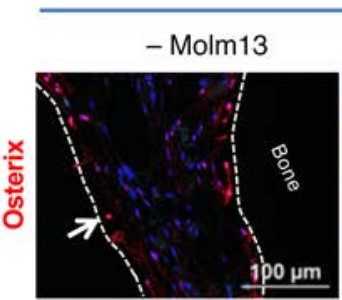

Osterix
C

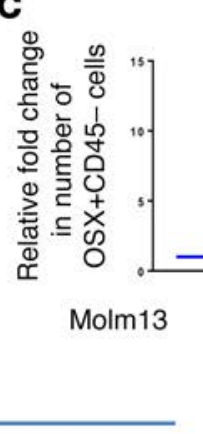

Osterix
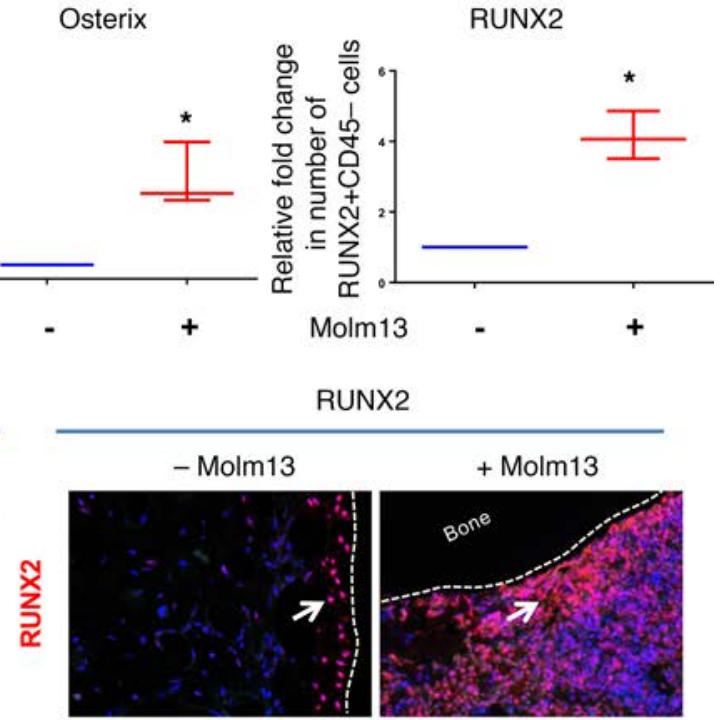

RUNX2

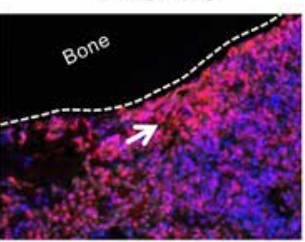

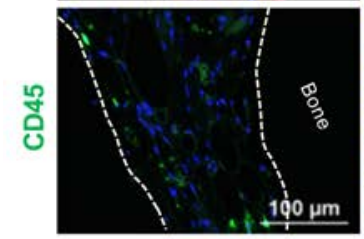
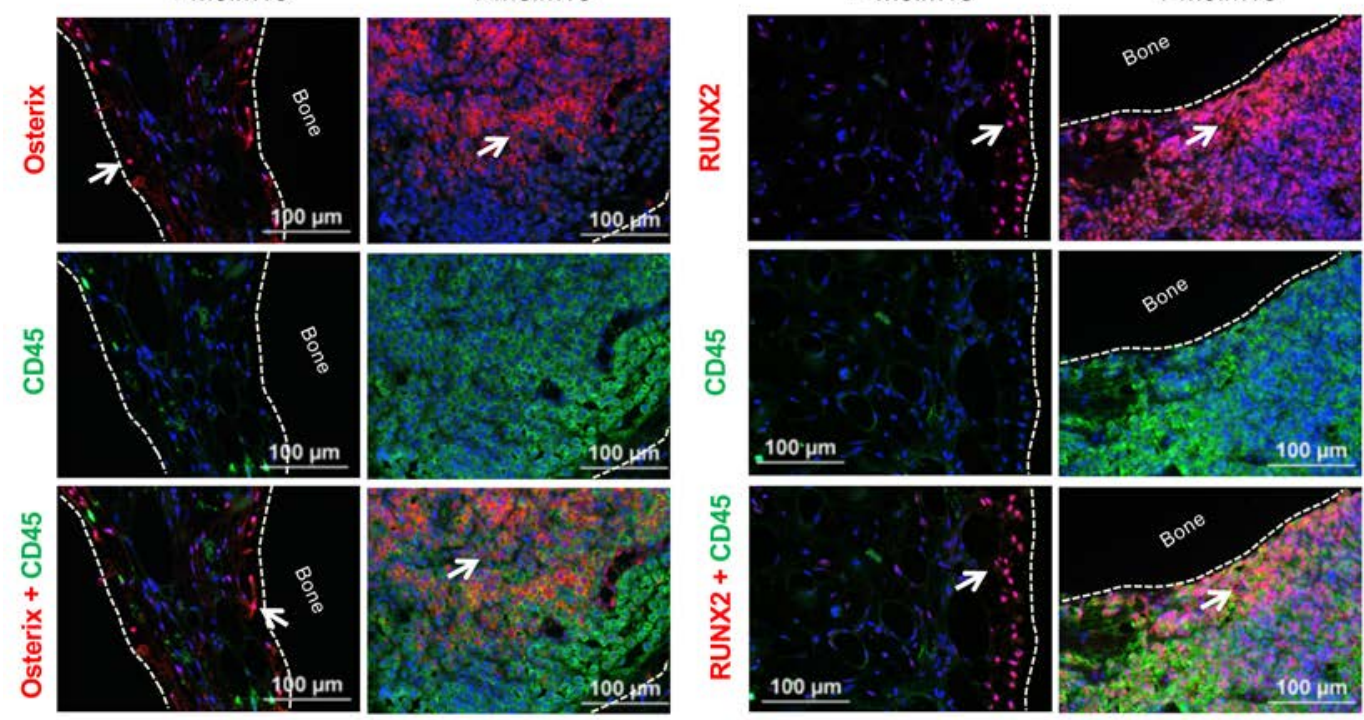

D

Normal BM

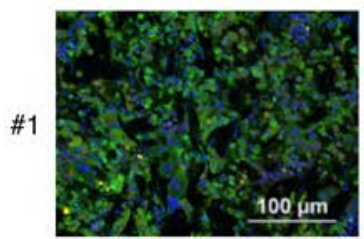

AML BM biopsy

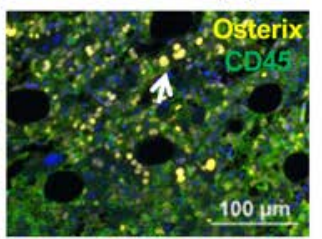

E

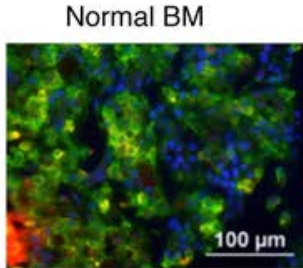

AML BM biopsy
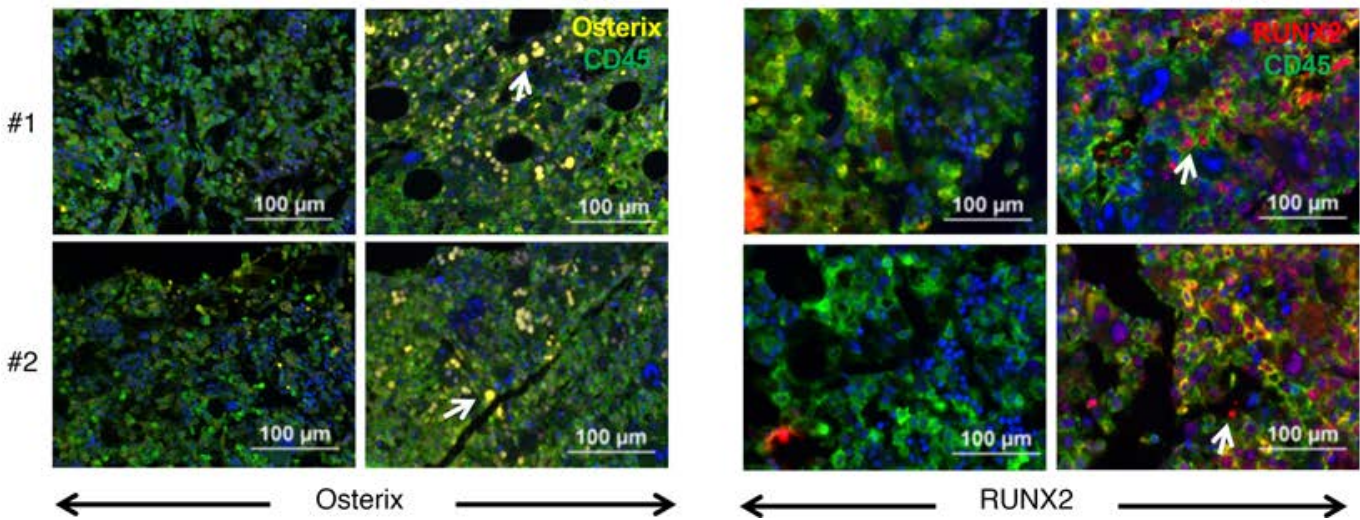

RUNX2

Figure 4. Human bone marrow implant model and patient AML-BM biopsy specimens validate AML-induced osteogenic differentiation in vivo. (A) Human femur-derived bone fragments were transplanted with Matrigel into the right flanks of NSG mice. Four weeks after implantation, vascularization of the HBMI bone fragments was confirmed by X-RAD 225 CX cone-beam micro-CT scan using the contrast agent AuroVist $15 \mathrm{~nm}$ injected intravenously before image acquisition. Images were imported to MicroView software for analysis and segmentation. This representative image shows neovascularization of the bone fragment. Arrow points towards a mouse blood vessel leading to HBMI. (B) Bone fragments from HBMI mice transplanted (right) or not transplanted (left) with Molm13 cells were subjected to IHC staining for CD45 and osterix or CD45 and RUNX2 4 weeks after transplantation. The Opal multiplex system was used for signal amplification. CD45 was labeled with fluorescein (green), and RUNX2 and osterix were developed with Cy5 label (red). The nuclei were stained with DAPI (blue). Arrow points towards cells expressing osterix or RUNX2. Scale bar: $100 \mu \mathrm{m}$. (C) Quantification of osterix ${ }^{+}$or RUNX2+ stromal cells from B. The absolute numbers of osterix ${ }^{+} \mathrm{CD}^{+} 5^{-}$or RUNX2+CD45- stromal cells from HBMI mice transplanted or not transplanted with Molm13 cells were quantitated by inForm image analysis software. Immunohistochemical analysis was performed for osterix (D) and RUNX2 (E) in normal (left) or AML patient-derived (right) BM specimens. The Opal multiplex system was used for signal amplification. CD45 staining was developed with fluorescein (green), osterix staining was developed with Cy3 (yellow), and RUNX2 staining was developed with Cy5 (red). Scale bar: $100 \mu \mathrm{m}$. Unpaired Student's $t$ test was used to test the statistical difference between the 2 groups $\left({ }^{*} P<\right.$ 0.05 ). Arrow points towards cells expressing osterix or RUNX2. 
A

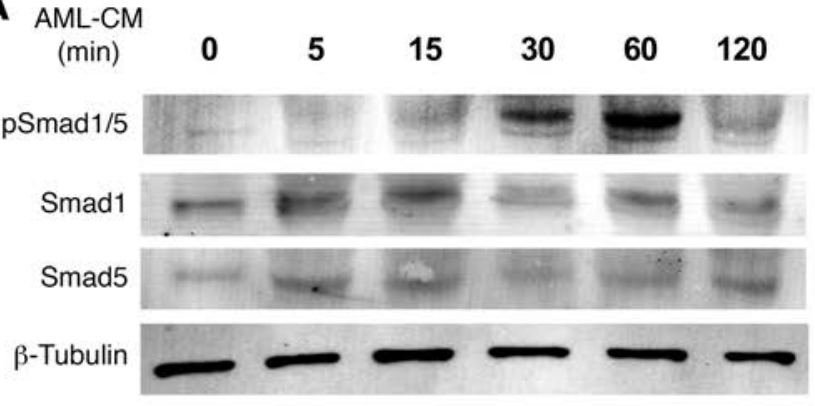

B

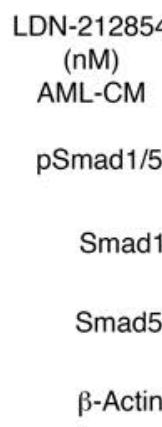

C

\section{RUNX2}

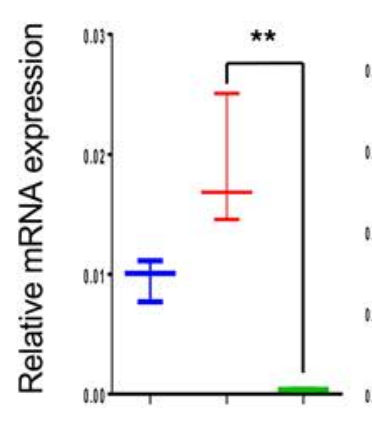

OCI-AML3

LDN-212854

$(1 \mu \mathrm{M})$

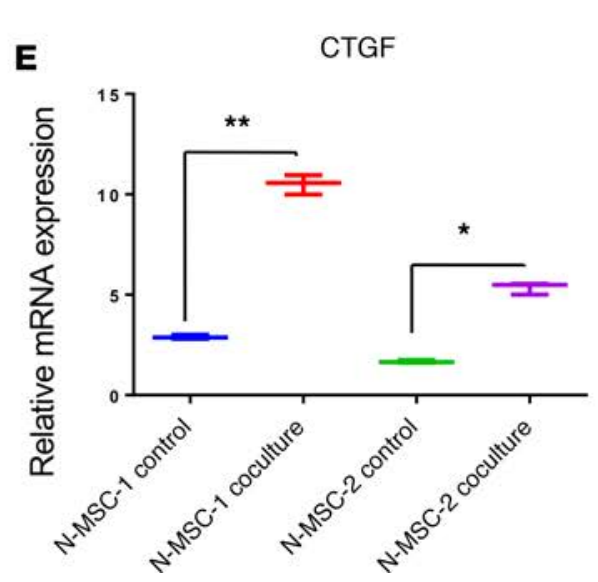

D
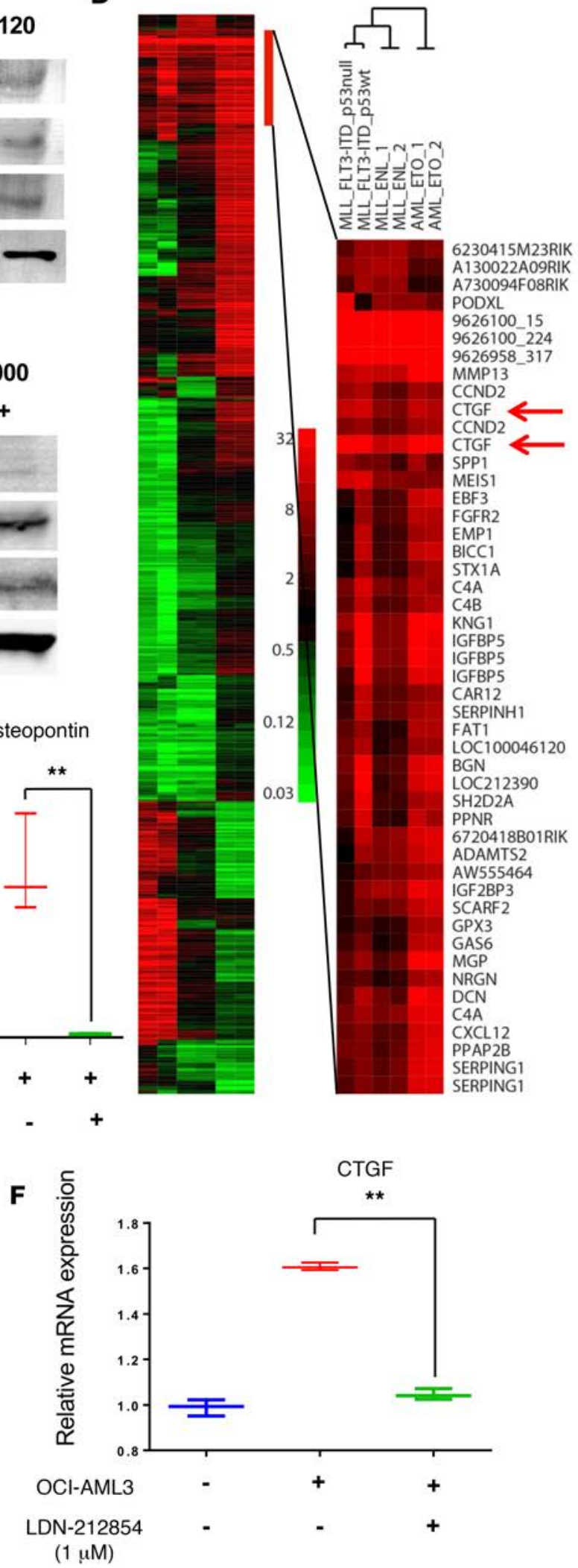

Figure 5. BMP signaling mediates AML-induced osteogenic differentiation and induces CTGF expression in MSCs. (A) Immunoblotting was used to detect total and phosphorylated Smad1/5 in MSCs cultured with or without OCI-AML3-conditioned medium at indicated time points. (B) Immunoblotting also was used to detect total and pSmad1/5 in MSCs cultured with or without AML-CM in the presence or absence of type-1 BMP inhibitor LDN-212854 at indicated concentrations. $\beta$-Tubulin and $\beta$-actin served as loading controls. Data shown are representative of 3 independent experiments. (C) N-MSCs ( $2 \times$ $10^{5}$ ) were cultured in OCI-AML3-conditioned medium (OCI-AML3) with or without LDN-212854 for 5 days. mRNA expression of osteoprogenitor-associated 
genes, RUNX2, osterix, and osteopontin, in the N-MSCs was measured by qRT-PCR. GAPDH served as an equal loading control. (D) Subtracted heatmap of change in microarray-based gene expression by BM-MSCs from in vivo exposure to different AML genotypes. Genes selected were those in which at least two of the genotype samples differed from control MSCs by at least 8-fold. The color bar shows the fold increase from normal MSC. Genes upregulated by all AML genotypes are expanded to the right. (E) N-MSCs were cocultured with or without OCI-AML3 cells for 5 days. After incubation, the N-MSCs were isolated by FACS, and total RNA was extracted from these cells. CTCF mRNA expression was analyzed by qRT-PCR ( $n=2$ ). (F) N-MSCs were cultured in the presence or absence of OCI-AML3-conditioned medium with or without LDN-212854 for 5 days. CTCF mRNA expression was measured by qRT-PCR. One-way ANOVA was used for comparisons of 3 or more groups and unpaired Student's $t$ test was used for comparisons of 2 groups ( $P<0.05$, ${ }^{*} P<0.01$ versus control). Tukey's multiple comparison test was also performed for multiple data sets.

cells induce osteogenic differentiation in MSCs. These findings were validated in vivo using an HBMI bone model. Osteogenic markers, including osterix and RUNX2, were upregulated in stromal cells in HBMI with leukemia compared to stromal cells in HBMI without leukemia. AML patient-derived BM biopsy samples also revealed higher osterix and RUNX2 expression compared with healthy BM. Mechanistic studies identified that AML cells secret BMPs and activate Smad1/5 phosphorylation in MSCs to induce osteogenic differentiation and CTGF expression in MSCs. Overexpressing CTGF in transgenic mice enhanced AML engraftment. These data demonstrate that AML cells induce osteogenic differentiation in BM-MSCs to gain growth advantage through growth factors secreted by osteogenic cells.

We previously demonstrated that AML-MSCs lack the ability to differentiate into adipocytes (22). However, their ability to differentiate into other lineages, including osteogenic or chondrogenic lineages, is not known. Here, we show that leukemia cells alter the BM microenvironment by inducing osteogenic differentiation in MSCs. In our previous report, we studied the effect of CTGF on MSCs but not on leukemia (22). Downregulation of CTGF induces adipogenic differentiation upon stimulation in vitro and spontaneously in vivo. These data support our current report that upregulation of CTGF in MSCs favors osteogenic differentiation but downregulation favors adipogenic differentiation. In our previous paper, we reported that $\mathrm{AML}$ cells home to BM with preexisting adipocytes compared with BM with fewer adipocytes (22). These data suggest that adipocytes could influence in AML homing to BM and engraftment. However, herein we show that AML cells influence MSCs in the BM to differentiate into osteoprogenitor cells. BMPs derived from AML cells activate Smad1/5 in MSCs and induce osteogenic differentiation. Once the cells become osteoprogenitor cells, they secret several factors, including CTGF, which supports leukemia growth. Several recent reports also suggest that the osteogenic BM niche affects AML growth. A report from Scadden's lab showed that deletion of Dicer1 from osterix-expressing osteoprogenitor cells induced leukemia in mice (28). In another report, Kousteni's group suggests that activating $\beta$-catenin mutations in osteoblasts induce AML in mice (29). Very recently, Dong and Yu et al., reported that Ptpn11 mutations in osteoprogenitor and mesenchymal stem cells induced myeloproliferative neoplasm but that the same mutations in endothelial cells or mature osteoblasts did not induce this effect on myeloid cell compartment (30). These reports from different labs suggest that osteogenic niches support leukemia growth and disease progression.

Hanoun et al. reported that AML-BM contained higher numbers of osterix-expressing osteogenic cells than healthy BM in a syngeneic mouse model (31). Recent data also suggest osteoblast-mediated inhibition of AML growth (32). However, the authors used osteocalcin-positive mature osteoblasts in their model, which are different from osterix ${ }^{+} \mathrm{RUNX}^{+}$osteoprogenitor cells. These reports and our data demonstrate that AML cells induce and require an osteoprogenitor-rich niche, but not mature osteoblasts, for their expansion. In fact, mature osteoblasts are essential for normal hematopoiesis (33). In primary AML, the expression of both osterix and RUNX2 was substantially upregulated (Figure 4D). Taken together, our data and that of published reports demonstrate that AML cells prefer a preosteoblast/osteoprogenitor-rich niche for their growth and that they generate this niche in the BM microenvironment by inducing osteogenic differentiation in MSCs.

BMPs play a critical role in the induction of osteogenic differentiation during embryonic development (23). We found that AML-conditioned medium mediates Smad1 and -5 activation in MSCs. Recent reports indicate that BMPs are highly expressed in plasma samples from AML patients compared with those from healthy individuals $(14,15,34)$, supporting our findings. Although it is not entirely clear which BMP proteins are involved, BMP6 and BMP9 have been shown to have the highest osteogenic activity among their family members (BMP1-15) (11) because of their association with Wnt signaling (12). In addition, BMP9 has been shown to directly regulate CTGF expression in MSCs (16), suggesting that it plays a functional role in AML-induced osteogenic differentiation in MSCs. 
A

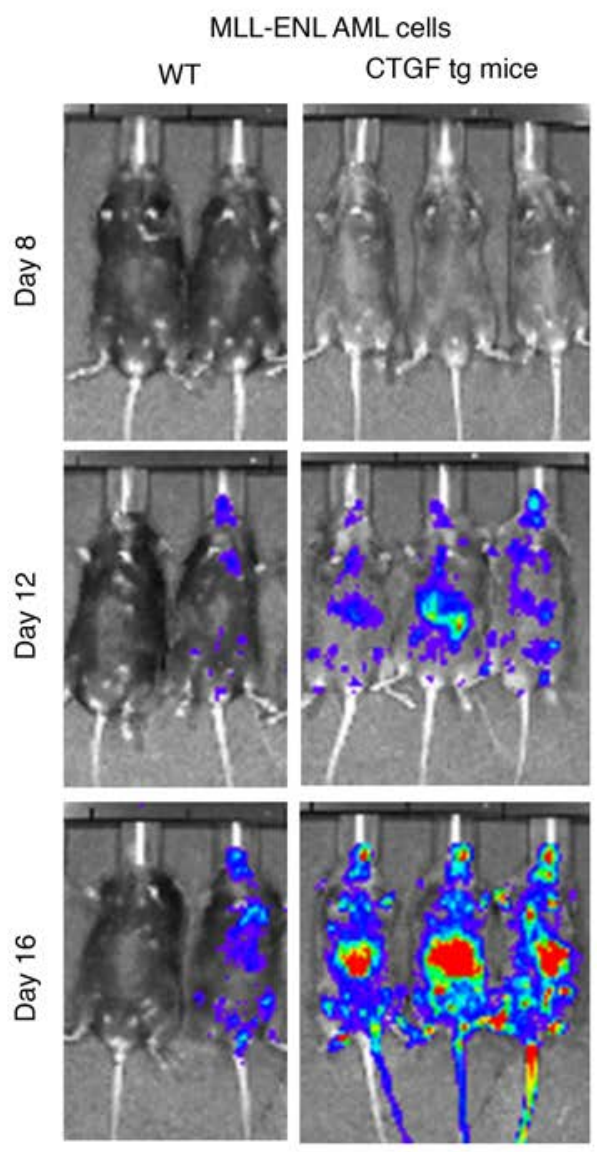

B

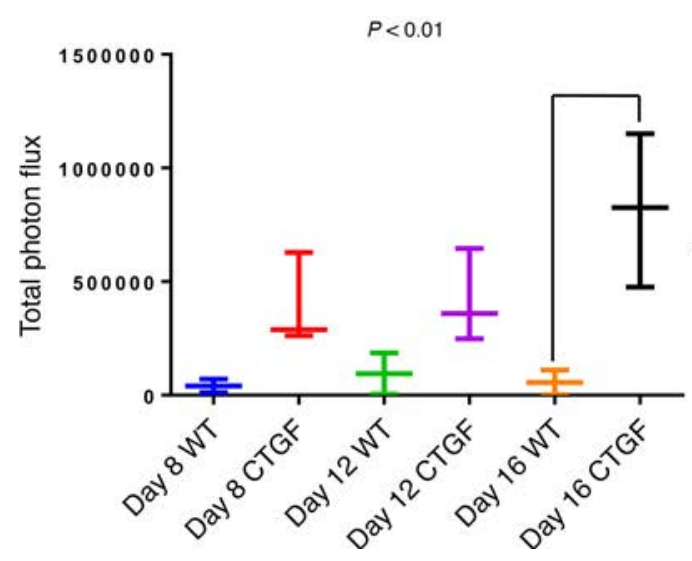

C

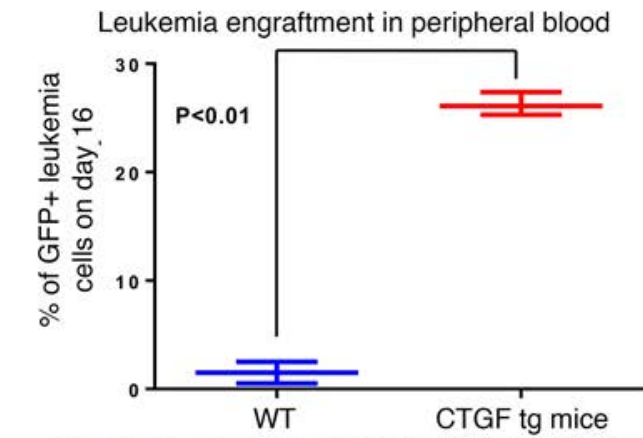

\section{$\stackrel{亠}{\Xi}$}
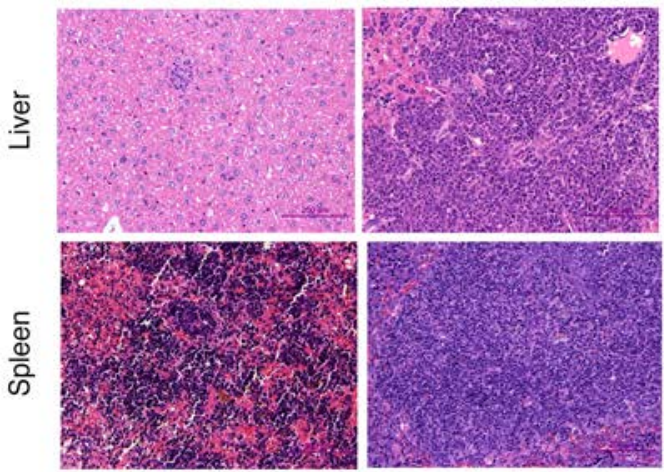

E

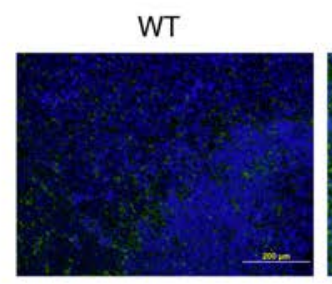

CTGF tg mice

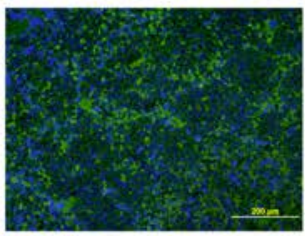

$\mathbf{F}$

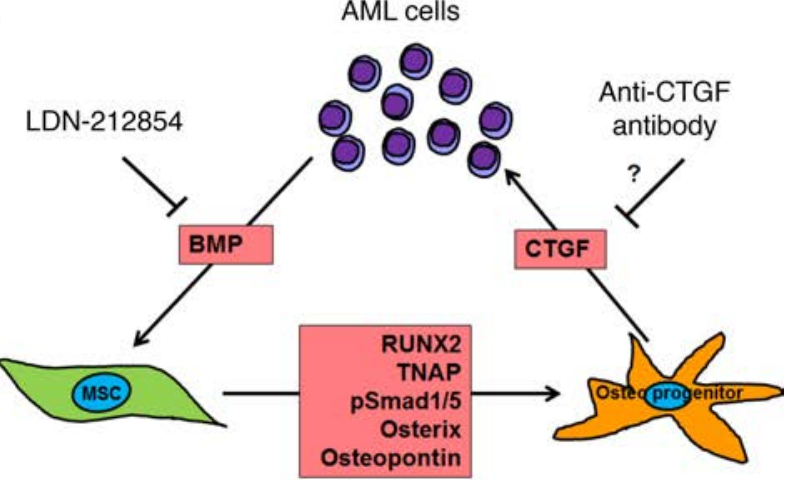

Figure 6. CTGF overexpression facilitates leukemia engraftment. (A) MLL-ENL-GFP-Nras-FFluci-p53 $3^{-1-}$ syngeneic mouse AML cells $\left(1 \times 10^{5}\right.$ per mouse) were transplanted intravenously into control (WT C57/B6; $n=3$ ) or Colla2-CTCF-transgenic mice (CTGF tg; $n=3$ ). Leukemic growth was measured by IVIS bioluminescence imaging at 8,12 , and 16 days after AML cell injection. (B) Total photon flux, representing the mean leukemia burden in the mice, was determined. (C) On day 16, peripheral blood mononuclear cells remaining after lysis of red blood cells were analyzed for GFP+ mouse leukemia cells by flow cytometry. (D) Sections of mouse spleen and liver from WT (left) or Col1a2-CTCF-transgenic mice were subjected to H\&E staining on day 16 after AML cell transplantation. Scale bar: $200 \mu \mathrm{m}$. (E) GFP immunofluorescence staining of spleens from WT mice (left) and Colla2-CTCF-transgenic mice (right) by the anti-GFP antibody on day 16 after AML cell transplantation. Scale bar: $200 \mu \mathrm{m}$. (F) Schematic representation of leukemia-stroma interaction in the BM microenvironment. Two-way ANOVA was used to test the data set in B and unpaired Student's $t$ test was used for the data set in $\mathbf{C}\left({ }^{* *} P<0.01,{ }^{* *} P<0.001\right.$ versus control). In addition, Tukey's multiple comparison test was also performed for the data set in $\mathbf{B}$. 
CTGF expression has been reported to be upregulated during osteogenic differentiation of mesenchymal cells (26). CTGF has been shown to be a prognostic factor in acute lymphoid leukemia, and inhibition of its expression by an anti-CTGF antibody (FG-3019) led to increased survival of leukemia-bearing mice (19). In addition, CTGF has been shown as a critical factor for normal hematopoietic stem cell (HSC) maintenance and it has been shown that HSCs can upregulate CTGF expression in stromal cells (35). However, the role of CTGF in AML has not been elucidated. Our findings, for the first time to our knowledge, define the role of CTGF in AML cell growth. Our data indicate that AML cells, but not normal $\mathrm{CD}^{+}{ }^{+}$cells, induce osteogenic differentiation through BMP/osterix/RUNX2-mediated signaling, leading to CTGF expression in MSCs, and that CTGF overexpression by stromal cells enhances leukemia engraftment in mice. Inhibition of BMP/CTGF-mediated signaling (Figure 6F) may inhibit AML growth and BM engraftment and could be developed into novel therapeutic concept in AML.

\section{Methods}

Isolation and culture of cells. MSCs were isolated from the BM of normal healthy donors undergoing BM harvest for use in allogeneic transplantation or AML patients. Each BM specimen was subjected to centrifugation ( $700 \mathrm{~g}$ for 20 minutes at $4^{\circ} \mathrm{C}$ ) over a Ficoll-Hypaque gradient (Sigma-Aldrich) to separate mononuclear cells. After centrifugation, the buffy coat layer was carefully extracted; resuspended in $\alpha$-MEM containing 10\% FBS, L-glutamine, $4 \%$ pooled human platelet lysate (36), and 1\% penicillin-streptomycin (MSC growth medium); and plated at an initial density of $5 \times 10^{5}$ cells $/ \mathrm{cm}^{2}$. After 3 days, the cultures were washed with PBS (Media Tech Inc.), and the remaining adherent cells were cultured until approximately $80 \%$ confluent. The cells were then subcultured at densities of 5,000-6,000 cells $/ \mathrm{cm}^{2}$. Only third or fourth passage cells were used for the experiments. AML cell lines, OCI-AML3 (gift from Mark Minden, Ontario Cancer Institute, Toronto, Canada) and Molm13 (Deutsche Sammlung von Mikroorganismen und Zellkulturen $\mathrm{GmbH}$ ), were cultured in RPMI medium (Media Tech Inc.) with $10 \% \mathrm{FBS}$ and $1 \%$ penicillin-streptomycin. CD $34^{+}$cells were isolated from human $\mathrm{CB}$ samples using magnet-assisted cell sorting (Miltenyi Biotech) as per the manufacturer's instructions.

Cell growth/cell cycle analysis. Cell growth was assessed by counting live cells on daily basis. AML- or N-MSCs ( $n=5$ each; $3 \times 10^{4}$ cells/well) were plated in 6-well cell culture dishes in MSC growth medium. Cells were trypsinized and counted daily for 6 days on an automated live-cell counter (Vi-CELL, Beckman Coulter). The data were analyzed in an Excel spreadsheet.

BrdU pulse labeling assay. AML- and N-MSCs ( $n=3$ each) were pulsed with $10 \mu \mathrm{M}$ BrdU solution for 3 hours in MSC growth medium. The cells were washed, subjected to trypsinization, and fixed in ice-cold ethanol overnight. Following fixation, DNA was denatured by incubating cells in $2 \mathrm{~N} \mathrm{HCl} / 0.5 \%$ Triton X-100 for 30 minutes at room temperature and then neutralized with0.1 $\mathrm{M} \mathrm{Na}_{2} \mathrm{~B}_{4} \mathrm{O}_{7}$. The cells were then incubated with anti-BrdU allophycocyanin (APC) for 30 minutes at room temperature. They were then washed and stained with PI solution ( $5 \mu \mathrm{g} / \mathrm{ml}$ PI, $0.5 \mathrm{mg} / \mathrm{ml}$ RNase, $0.5 \%$ Tween 20 , and $1 \%$ bovine serum albumin in PBS). The samples were then washed and measured on an LSR-II flow cytometer (Becton Dickinson Immunocytometry Systems). Cell cycle distribution was analyzed by FlowJo software (Tree Star Inc.).

Flow cytometry. Flow cytometry analysis of MSCs isolated from normal donor-derived and AML patient-derived BM specimens was performed as described before (22). MSCs isolated from normal donorderived and AML patient-derived BM specimens were subjected to trypsinization and washed once with PBS. The cells were then incubated for 20 minutes with $10 \mu 1$ of fluorochrome-conjugated antibodies. The following antibody conjugates were used: anti-CD45, anti-CD105, anti-CD140b, anti-CD146, antiCD73, anti-TNAP (clone W8B2), and anti-CD140a were conjugated with phycoerythrin (PE); anti-CD271 and anti-CD51 were conjugated with FITC; anti-CD44, anti-SUSD2, and anti-CD106 were conjugated with APC; and anti-CD31 was conjugated with APC/cyanine dye Cy7 (all purchased from BioLegend). Anti-CD90 antibody conjugated with APC-Alexa fluor 700 was purchased from Beckman Coulter. After incubation, the cells were washed once with PBS containing $0.5 \mu \mathrm{g} / \mathrm{ml}$ DAPI (to exclude dead cells) and analyzed on an LSR-II Flow Cytometer. Ten thousand events were acquired for each sample. The flow cytometric data were analyzed by FlowJo software.

Multilineage differentiation. For experiments related to osteogenic and adipogenic differentiation, we randomly selected AML and normal MSCs. Samples 5, 6, 11, and 22 from AML-MSCs and samples 31, 34, and 36 from normal MSCs (Supplemental Table 1) were used for differentiation induction or coculture experiments. Multilineage differentiation potential of the AML- and N-MSCs was investigated as described before $(22,37)$. 
To induce osteogenic differentiation, 10,000 MSCs isolated from normal donor- or AML patientderived BM were cultured with MSC growth medium (control) or with NH OsteoDiff medium (Miltenyi Biotec) for 21 days in 6-well dishes. The medium was replaced every 3 days. After 21 days, the cells were washed twice with PBS and fixed with $4 \%$ paraformaldehyde (PFA). To determine ALP activity, the cells were incubated with FAST BCIP/NBT substrate (Sigma-Aldrich) for 20 minutes at room temperature. Calcium deposition was analyzed by staining with $1 \%$ Alizarin Red S (Spectrum) for 20 minutes at room temperature. After the cells were washed, they were photographed by a Nikon Coolpix-950 camera attached to a Nikon-TMS light microscope.

To measure adipocyte differentiation, MSCs isolated from normal donor- or AML patient-derived BM were cultured in MSC growth medium (control) or adipocyte differentiation medium containing DMEM, $1 \mu \mathrm{M}$ dexamethasone, $50 \mu \mathrm{M}$ indomethacin, $500 \mathrm{nM}$ IBMX, $5 \mu \mathrm{g} / \mathrm{ml}$ insulin, and 10\% fetal calf serum (FCS) in a 12-well cell culture dish. The medium was replaced every 3 days. After $21-28$ days, the formation of adipocytes was evaluated by fixing cells with 4\% PFA and staining with Oil Red O dye (Sigma-Aldrich) for 15 minutes at room temperature.

To induce differentiation into the chondrocyte lineage, the cells were incubated in ChondroDiff medium (Miltenyi Biotec). Briefly, cells were washed once with PBS and plated into a u-bottom 96-well plate with 250,000 MSC cells/well. The medium was replaced every 3 days. After incubation for 28 days, the resulting cell pellets were fixed with $10 \%$ formalin, embedded in paraffin, and cut into $5-\mu \mathrm{m}$ sections. Following deparaffinization and hydration, the sections were incubated with $1 \%$ Alcian Blue 8GX (w/v) solution (Sigma-Aldrich) in 3\% acetic acid for 30 minutes at room temperature. The slides were washed in $3 \%$ acetic acid and then in distilled water. Photographs were taken by an Olympus DP72 digital camera attached to a BX43 microscope (Olympus America Inc).

Alkaline phosphatase and mineralization quantification. ALP activity was quantified by using the colorimetric Alkaline Phosphatase Assay Kit (Abcam) as per the manufacture's instructions. Mineral deposition by cultured cells was quantified directly from Alizarin Red S staining as previously described (38). Briefly, cells were trypsinized, washed once with PBS, and subjected to lysis in assay buffer. The assay was performed by adding $5 \mathrm{mM}$ p-nitrophenol phosphate substrate and sample lysis buffer to cells in a 96-well flat-bottom plate. A standard curve was prepared by diluting $1 \mathrm{mM}$ p-nitrophenol phosphate stock with appropriate amounts of assay buffer. The standards ranged from 0.015 to $0.15 \mu \mathrm{mol} / \mathrm{ml}$. After incubation for 1 hour at room temperature, absorbance was measured at $405 \mathrm{~nm}$ by using Infinite 200 Pro (Tecan). A standard curve of absorbance versus p-nitrophenol concentration was plotted and used to determine the amount of p-nitrophenol generated by each sample, representing ALP activity.

The dye was extracted by incubating cells with $10 \%(\mathrm{v} / \mathrm{v})$ acetic acid for 30 minutes at room temperature with shaking. The monolayer was scraped with a cell scraper and transferred to a $1.5-\mathrm{ml}$ microcentrifuge tube. After vortexing for 30 seconds, the lysate was sealed with parafilm, heated to $85^{\circ} \mathrm{C}$ for 10 minutes, and immediately cooled on ice for at least 5 minutes. The lysate was then subjected to centrifugation at 20,000 g for 15 minutes. The supernatant was transferred to a new tube and neutralized with $10 \%(\mathrm{v} / \mathrm{v})$ ammonium hydroxide to reach $\mathrm{pH}$ between 4.1 and 4.5 before aliquoting to a 96 -well plate. The standards ranged from 0.05 to $0.4 \mu \mathrm{mol} / \mathrm{ml}$. Absorbance was read at $405 \mathrm{~nm}$ with an Infinite 200 Pro.

Quantitative RT-PCR. Unless otherwise mentioned, all qRT-PCR analyses were performed as SYBRgreen PCR analysis. Total RNA was extracted by the standard Trizol-based method described elsewhere (22). The cDNA was prepared by using the Improm-II reverse transcriptase system (Promega). The SYBRgreen PCR primer sequences and taqman assays used for experiments in this report are listed in Supplemental Table 2 and Supplemental Table 3, respectively. The Fast-SYBR-green or Fast-taqman master consisting of DNA polymerase and a nucleotide mix was purchased from Applied Biosystems (now ThermoFisher Scientific). An HT900 real-time PCR instrument (ThermoFisher Scientific) was used to perform the PCR reaction and acquisition of signal amplification.

Human BM implant mouse model. Human femur heads were obtained from leukemia-free patients undergoing hip replacement surgery at St. Luke's Hospital (Houston, Texas, USA). NOD/SCID/IL-2r $\gamma^{\text {null }}$ (NSG) mice were purchased from the Jackson Laboratory. Eight- to 10-week-old NSG mice were surgically implanted with the fresh human femur fragments as a model of normal human BM. The mice were irradiated (150 Gy) the day before the surgery, and the fresh bone fragments were irradiated (250 Gy) the day of the surgery to avoid graft-versus-host disease in the recipient mice. The implantation was done under sterile conditions. Before the surgery, animals were anesthetized with isoflurane (2.5\%) and the mice were shaved 
on the dorsal side until the skin was clearly visible. Two 1-cm incisions were made in the right dorsal flank of each mouse using a sterile sharp scalpel, and human bone fragments immersed in Matrigel (ECMatrix, Millipore) were carefully implanted subcutaneously into these incisions. The incisions were then closed with surgical clips. The animals were maintained on a Bactrim diet (Harlan Laboratories Inc.) for up to 2 weeks following surgery. The bone implants were allowed to engraft in the mice for 4 to 12 weeks. For the leukemia engraftment assays, leukemia cells were introduced through the tail vein 4 weeks after bone implantation. The experiment was repeated 3 times using 5 mice per group.

CT imaging. The vascularization of the newly implanted human bone fragments in the HBMI mice was assessed by imaging on a X-RAD 225 Cx cone-beam micro-CT (Precision X-Ray) using the commercially available contrast agent AuroVist $15 \mathrm{~nm}$ (Nanoprobes) as described elsewhere (39). The injected doses of blood-pool agents were selected following the instructions provided by the manufacturer. Both before and after contrast scans were taken. The contrast material was injected continuously via a polyethylene catheter though the tail vein in a $200-\mu 1$ solution per $25 \mathrm{~g}$ mouse ( $40 \mu \mathrm{g} /$ mouse), with an injection time of $2 \mathrm{~min}$ utes. Scan tube voltage was $40 \mathrm{kVp}$ and current was $5 \mathrm{~mA}$. For each scan, 360 projections were acquired at 1-degree increments and reconstructed using a filtered back-projection algorithm, resulting in an image volume with isotropic $150-\mu \mathrm{m}$ voxels. Images were imported into the MicroView system (Parallax Innovations) for analysis and segmentation.

Bone generation by human bone implants. New bone generation was assessed as described elsewhere (40). Briefly, the animals were injected intravenously with the targeted fluorescent in vivo bisphosphonate imaging agent OsteoSense 750EX (PerkinElmer; $100 \mu \mathrm{l} /$ mouse), which images bone growth, reabsorption, remodeling, and microcalcifications (41). As a control, mice without human bone implants were also injected with OsteoSense 750. The mice were imaged by using the IVIS (PerkinElmer) system, and the imaging data were analyzed by Living Image software (PerkinElmer).

MSC generation from human bone implants. The HBMI mice were killed 4 weeks after bone implantation, and the bone implants were removed and cut into $0.5-\mathrm{cm}^{3}$ cubes. These pieces of recovered bone were washed once with PBS and incubated in a combination of dispase $(0.5 \mathrm{mg} / \mathrm{ml}$; Stem Cell Technologies $)$ and collagenase $\left(1 \mathrm{mg} / \mathrm{ml}\right.$; Sigma-Aldrich) at $37^{\circ} \mathrm{C}$ for 60 minutes in a shaking incubator. After incubation, the bone pieces were washed twice with DMEM containing 10\% FBS. The HBMI-derived cells were subjected to red cell lysis buffer (Sigma-Aldrich) for 10 minutes to remove any residual erythrocytes. After incubation, the cells were washed twice with PBS and plated in MSC growth medium. The MSCs generated were isolated by FACS using anti-human CD140b and anti-human CD90 markers to exclude any mouse-derived MSCs.

Conditioned medium incubation and Western blotting. To investigate the induction of BMP signaling in MSCs by leukemic cells, AML-conditioned medium was prepared by seeding OCI-AML3 cells $\left(0.3 \times 10^{6}\right.$ cells $/ \mathrm{ml}$ ) in RPMI medium with $2 \%$ FCS. The cells were harvested on day 4 , and the supernatants were collected and filtered through $0.2-\mu \mathrm{M}$ membranes (Millipore). N-MSCs, pretreated for 1 hour with BMP type-1 receptor inhibitor LDN-212854 (Sigma-Aldrich) at different concentrations were incubated in the resulting supernatants. Cells were then subjected to lysis at a density of $3 \times 10^{5} / 50 \mu 1$ in protein lysis buffer (Mammalian Cell-PE LB Buffer, G Biosciences; 2' Laemmli Sample Buffer, Bio-Rad) supplemented with 1:200 protease and phosphatase inhibitor cocktail (Cell Signaling Technologies) and 1:200 $\beta$-mercaptoethanol (Sigma-Aldrich). Cell lysates were loaded onto a 10\% polyacrylamide gel (Bio-Rad) for electrophoresis, and the proteins were then transferred to Immobilon-FL membranes (Millipore). The membranes were incubated with rabbit anti-human phospho (p) Smad1/5 or anti-Smad1 or anti-Smad5 antibodies (all from Cell Signaling Technologies) and mouse anti-human $\beta$-tubulin antibody (Sigma-Aldrich) overnight at $4^{\circ} \mathrm{C}$. They were then washed and incubated with donkey anti-rabbit or anti-mouse IgG secondary antibody conjugated with Alexa fluor 680 or Alexa fluor 800 (both from LI-COR Biosciences). The membranes were washed again and scanned using the Odyssey fluorescence imaging system (LI-COR Biosciences).

Leukemia engraftment. To investigate leukemia engraftment in human bone implants, we transplanted Molm13 cells $\left(10^{6}\right)$ expressing GFP and firefly luciferase through the tail vein in a group of HBMI mice ( $n$ $=5)$. Another group of HBMI mice $(n=5)$ that did not receive leukemia cells served as controls. The leukemia engraftment was measured weekly by IVIS whole-body imaging (Caliper Life Sciences) and peripheral blood analysis by flow cytometry. Human leukemia engraftment in the peripheral blood was assessed by positive staining for anti-human CD45-PE antibody (Biolegend) and negative staining for anti-mouse CD45-APC. Leukemia engraftment in HBMI mice was also assessed by H\&E staining (Sigma-Aldrich) and by IHC staining for anti-human CD45 and firefly luciferase. 
The Colla2-CTGF-transgenic mice used in this study have been described previously (42). Briefly, the murine homolog of CTGF (Fisp-12) was cloned into a vector containing the 6-kb enhancer and minimal promoter of the murine Colla2 gene, an IRES-lacZ reporter, and the murine protamine polyA signal. Transgenic mice were produced by the standard method of pronuclear injection of linear DNA into a fertilized mouse egg. Genotyping was performed by PCR for the lacZ reporter on DNA extracted from ear biopsies. Mouse hematopoietic cells transduced with MLL-ENL-GFP Nras-FFluci p53 ${ }^{-/-}$(gift of Scott Lowe) were introduced into a group of CTGF-transgenic and WT (C57/B6) mice via tail vein. Bioluminescent imaging was performed at different time points by injecting D-luciferin (4 $\mathrm{mg} / \mathrm{mouse}$ in $100 \mu \mathrm{l}$ ). The leukemia engraftment was measured weakly by whole-body imaging using IVIS (Caliper Life Sciences) and peripheral blood by flow cytometry analysis. Leukemia engraftment in the peripheral blood was assessed by $\mathrm{GFP}^{+}$leukemia cells. Leukemia engraftment was also assessed by immunohistochemical staining using anti-GFP antibody.

Immunohistochemical analysis. After substantial human leukemia engraftment $(>30 \%$ circulating human $\mathrm{CD} 45^{+}$cells), mice were killed and the human bone implants were removed and fixed in $4 \%$ PFA. The bone fragments were decalcified and the remaining tissues were embedded in paraffin and cut into thin sections $(5 \mu \mathrm{m})$ onto glass slides using a microtome. The slides were stained with $\mathrm{H} \& \mathrm{E}$ and analyzed by light microscopy. For IHC staining, the tissue sections were deparaffinized and gently boiled in $1^{\prime}$ antigen retrieval buffer (DAKO) in a microwave oven for 15 minutes at 20\% power. The slides were then washed and incubated for 10 minutes in serum-free blocking solution (DAKO) and then for 1 hour with the primary antibody. The tissue sections were washed 3 times with TBST and stained with horseradish peroxidase-conjugated species-specific secondary antibodies. The slides were then washed again and developed with Opal fluorescein and Cy5 reagents (Perkin Elmer) per the manufacturer's guidelines. The slides were imaged with a Vectra multispectral imaging system (Perkin Elmer), and the data were processed by inForm image analysis software (Perkin Elmer). Leukemia engraftment in CTGF-transgenic mice was analyzed by using Alexa fluor 488-conjugated anti-mouse secondary antibody (Life Technologies) instead of Opal.

Gene expression profiling. To investigate differences in gene expression among AML-associated MSCs and healthy MSCs, murine AML cells carrying different gene deletions/rearrangements were transplanted in C57/B6 mice. These primary leukemia cells were generated by introducing the desired oncogenic translocations, including p53-knockout C57BL/6 mice (AML1/ETO9a, MLL/ENL, MLL/ENL+FLT3-ITD) or p53 wt C57BL/6 mice (MLL/ENL+FLT3-ITD p53 wt). In vivo expanded genetically modified leukemia cells were transplanted into 10 mice ( 6 to 8 week old) by tail vein injection of $1 \times 10^{6}$ cells/recipient. PBS was injected in the control group. Mice were sublethally irradiated prior to the injections to favor a uniform disease onset in recipient animals. Leukemia engraftment and burden were subsequently monitored by bioluminescence imaging (IVIS system; Xenogen/Caliper Life Sciences).

Transplanted leukemia was allowed to progress for 2 (MLL/ENL and MLL/ENL+FLT3-ITD), 3 (AML1/ETO9a), or 4 weeks (MLL/ENL+FLT3-ITD p53 wt) according to disease burden. At this time, mice were sacrificed and BM-MSCs from mouse BM were isolated by FACS using a combination of the following cell surface markers: Ter119-, CD31-, CD45-, CD105 ${ }^{+}, \mathrm{Sca}^{+}, \mathrm{CD} 106^{+}$, and PDGF-R $\alpha^{+}$ (CD140) $(43,44)$. Gene expression analysis was performed as described before (22). Briefly, total RNA was extracted from BM-MSCs isolated from mice using the RNAqueous kit (Ambion). After confirmation of RNA quality using a Bioanalyzer 2100 instrument (Agilent), 300 ng total RNA was amplified and biotin-labeled through an Eberwine procedure using an Illumina TotalPrep RNA Amplification kit (Ambion) and hybridized to Illumina HT12 version 4 mouse whole-genome arrays.

Statistics. Differences in TNAP expression were compared by subjecting MFI \pm SEM values from $\mathrm{N}$ - or AML-MSCs to ANOVA test using the Prism software. This method was also used to compare data from RT-PCR gene expression studies and enzyme activity assays. $P$ values of less than 0.05 were considered statistically significant. One-way or two-way ANOVA was used for comparisons of 3 or more groups, and unpaired 2-tailed Student's $t$ test was used for comparisons of 2 groups. In addition, Dunnett's or Tukey's multiple comparison test was used for multiple groups. For comparison of osterix ${ }^{+} \mathrm{CD} 45^{-}$or RUNX2 ${ }^{+} \mathrm{CD} 45^{-}$stromal cells in HBMI mice stimulated with leukemia cells or not stimulated, cell-level marker intensity was dichotomized relative to the median intensity in the population. Thus, every cell was assigned high or low marker status based on its intensity relative to the median. For each of the markers (osterix, CD45, and Runx2), the number of cells that were in each 
subgroup (Runx2 ${ }^{+} \mathrm{CD} 45^{+}$; Runx2 ${ }^{+} \mathrm{CD} 45^{-}$; Runx2-CD45+; and Runx2-CD45-) were counted. Similarly, for the Osterix ${ }^{+}$and $\mathrm{CD} 45^{+}$populations, a test of proportions was used to measure the difference in the population of Runx $2^{+} \mathrm{CD} 45^{-}$cells between the 3 groups relative to the control (without Molm13). This procedure was repeated for the osterix ${ }^{+} \mathrm{CD} 45^{-}$comparison as well.

Study approval. All experiments performed with the mice were in compliance with the standards of care of the MD Anderson Cancer Center Institutional Animal Care and Use Committee as well as in accordance with a protocol approved by the MD Anderson Cancer Center Institutional Animal Care and Use Committee. All BM and bone donors provided written informed consent. Studies involving human materials were conducted according to a protocol approved by the Institutional Review Boards of St. Luke's and MD Anderson Cancer Center.

\section{Author contributions}

VLB conceptualized and planned the study, conducted experiments, interpreted the data, and wrote the manuscript. PML and JCS performed the experiments and analyzed the data. KN, BY, XZ, SS, TM, VR, KAM, and XL performed the experiments. ES provided patient samples. ZW performed gene expression array experiments and the data was analyzed by RJ and RED. AR analyzed statistical data. GA provided patient samples. MK interpreted the data. MAH and CWC provided human bone samples. CBR provided samples and interpreted the data. MA planned the study and interpreted the data.

\section{Acknowledgments}

This work was supported in part by the MD Anderson Cancer Center Support Grant CA016672, the Paul and Mary Haas Chair in Genetics (to MA), the Rolanette and Berdon Lawrence Research Award, a Leukemia SPORE career development award, an MD Anderson Cancer Center Institutional Research Grant (to VLB), and National Institutes of Health grant R01CA155056 (to MK).

Address correspondence to: Venkata Lokesh Battula or Michael Andreeff, Section of Molecular Hematology and Therapy, Departments of Leukemia, University of Texas MD Anderson Cancer Center, 1515 Holcombe Boulevard, Unit 425, Houston, Texas, 77030 USA. Phone: 713.563.2227; Email: vbattula@ mdanderson.org (V.L. Battula). Phone: 713.792.7261; Email: mandreef@mdanderson.org (M. Andreeff).

1. Colmone A, Amorim M, Pontier AL, Wang S, Jablonski E, Sipkins DA. Leukemic cells create bone marrow niches that disrupt the behavior of normal hematopoietic progenitor cells. Science. 2008;322(5909):1861-1865.

2. Morrison SJ, Spradling AC. Stem cells and niches: mechanisms that promote stem cell maintenance throughout life. Cell. 2008;132(4):598-611.

3. Konopleva MY, Jordan CT. Leukemia stem cells and microenvironment: biology and therapeutic targeting. J Clin Oncol. 2011;29(5):591-599.

4. Becker PS. Dependence of acute myeloid leukemia on adhesion within the bone marrow microenvironment. ScientificWorldJournal. 2012;2012:856467.

5. Konopleva M, Konoplev S, Hu W, Zaritskey AY, Afanasiev BV, Andreeff M. Stromal cells prevent apoptosis of AML cells by up-regulation of anti-apoptotic proteins. Leukemia. 2002;16(9):1713-1724.

6. Nwajei F, Konopleva M. The bone marrow microenvironment as niche retreats for hematopoietic and leukemic stem cells. Adv Hematol. 2013;2013:953982.

7. Short B, Brouard N, Occhiodoro-Scott T, Ramakrishnan A, Simmons PJ. Mesenchymal stem cells. Arch Med Res. 2003;34(6):565-571.

8. Friedman MS, Long MW, Hankenson KD. Osteogenic differentiation of human mesenchymal stem cells is regulated by bone morphogenetic protein-6. J Cell Biochem. 2006;98(3):538-554.

9. Morsczeck C. Gene expression of runx2, Osterix, c-fos, DLX-3, DLX-5, and MSX-2 in dental follicle cells during osteogenic differentiation in vitro. Calcif Tissue Int. 2006;78(2):98-102.

10. Westhrin M, Xie M, Olderøy MØ, Sikorski P, Strand BL, Standal T. Osteogenic differentiation of human mesenchymal stem cells in mineralized alginate matrices. PLoS ONE. 2015;10(3):e0120374.

11. Kang Q, et al. Characterization of the distinct orthotopic bone-forming activity of 14 BMPs using recombinant adenovirus-mediated gene delivery. Gene Ther. 2004;11(17):1312-1320.

12. Tang N, et al. BMP-9-induced osteogenic differentiation of mesenchymal progenitors requires functional canonical Wnt/ beta-catenin signalling. J Cell Mol Med. 2009;13(8B):2448-2464.

13. Mizrahi O, et al. BMP-6 is more efficient in bone formation than BMP-2 when overexpressed in mesenchymal stem cells. Gene Ther. 2013;20(4):370-377.

14. Raymond A, et al. A role for BMP-induced homeobox gene MIXL1 in acute myelogenous leukemia and identification of type I BMP receptor as a potential target for therapy. Oncotarget. 2014;5(24):12675-12693.

15. Crispino JD, Le Beau MM. BMP meets AML: induction of BMP signaling by a novel fusion gene promotes pediatric acute leu- 
kemia. Cancer Cell. 2012;22(5):567-568.

16. Luo Q, et al. Connective tissue growth factor (CTGF) is regulated by Wnt and bone morphogenetic proteins signaling in osteoblast differentiation of mesenchymal stem cells. J Biol Chem. 2004;279(53):55958-55968.

17. Lee CH, Shah B, Moioli EK, Mao JJ. CTGF directs fibroblast differentiation from human mesenchymal stem/stromal cells and defines connective tissue healing in a rodent injury model. J Clin Invest. 2010;120(9):3340-3349.

18. Sala-Torra O, et al. Connective tissue growth factor (CTGF) expression and outcome in adult patients with acute lymphoblastic leukemia. Blood. 2007;109(7):3080-3083

19. Lu H, et al. Targeting connective tissue growth factor (CTGF) in acute lymphoblastic leukemia preclinical models: anti-CTGF monoclonal antibody attenuates leukemia growth. Ann Hematol. 2014;93(3):485-492.

20. Ochiai-Shino $\mathrm{H}$, et al. A novel strategy for enrichment and isolation of osteoprogenitor cells from induced pluripotent stem cells based on surface marker combination. PLoS ONE. 2014;9(6):e99534.

21. Battula VL, et al. Isolation of functionally distinct mesenchymal stem cell subsets using antibodies against CD56, CD271, and mesenchymal stem cell antigen-1. Haematologica. 2009;94(2):173-184.

22. Battula VL, et al. Connective tissue growth factor regulates adipocyte differentiation of mesenchymal stromal cells and facilitates leukemia bone marrow engraftment. Blood. 2013;122(3):357-366.

23. Hogan BL. Bone morphogenetic proteins: multifunctional regulators of vertebrate development. Genes Dev. 1996;10(13):1580-1594.

24. Mohedas AH, Xing X, Armstrong KA, Bullock AN, Cuny GD, Yu PB. Development of an ALK2-biased BMP type I receptor kinase inhibitor. ACS Chem Biol. 2013;8(6):1291-1302.

25. Zuber J, et al. Mouse models of human AML accurately predict chemotherapy response. Genes Dev. 2009;23(7):877-889.

26. Safadi FF, et al. Expression of connective tissue growth factor in bone: its role in osteoblast proliferation and differentiation in vitro and bone formation in vivo. J Cell Physiol. 2003;196(1):51-62.

27. Arnott JA, et al. The role of connective tissue growth factor (CTGF/CCN2) in skeletogenesis. Crit Rev Eukaryot Gene Expr. 2011;21(1):43-69.

28. Raaijmakers $\mathrm{MH}$, et al. Bone progenitor dysfunction induces myelodysplasia and secondary leukaemia. Nature. 2010;464(7290):852-857.

29. Kode A, et al. Leukaemogenesis induced by an activating $\beta$-catenin mutation in osteoblasts. Nature. 2014;506(7487):240-244.

30. Dong L, et al. Leukaemogenic effects of Ptpn11 activating mutations in the stem cell microenvironment. Nature. 2016;539(7628):304-308.

31. Hanoun M, et al. Acute myelogenous leukemia-induced sympathetic neuropathy promotes malignancy in an altered hematopoietic stem cell niche. Cell Stem Cell. 2014;15(3):365-375.

32. Krevvata M, et al. Inhibition of leukemia cell engraftment and disease progression in mice by osteoblasts. Blood. 2014;124(18):2834-2846.

33. Calvi LM, et al. Osteoblastic cells regulate the haematopoietic stem cell niche. Nature. 2003;425(6960):841-846.

34. Grcević D, Marusić A, Grahovac B, Jaksić B, Kusec R. Expression of bone morphogenetic proteins in acute promyelocytic leukemia before and after combined all trans-retinoic acid and cytotoxic treatment. Leuk Res. 2003;27(8):731-738.

35. Istvánffy R, et al. Stroma-derived connective tissue growth factor maintains cell cycle progression and repopulation activity of hematopoietic stem cells in vitro. Stem Cell Reports. 2015;5(5):702-715.

36. Schallmoser K, Strunk D. Preparation of pooled human platelet lysate (pHPL) as an efficient supplement for animal serum-free human stem cell cultures. J Vis Exp. 2009;(32):1523.

37. Battula VL, et al. Human placenta and bone marrow derived MSC cultured in serum-free, b-FGF-containing medium express cell surface frizzled-9 and SSEA-4 and give rise to multilineage differentiation. Differentiation. 2007;75(4):279-291.

38. Gregory CA, Gunn WG, Peister A, Prockop DJ. An Alizarin red-based assay of mineralization by adherent cells in culture: comparison with cetylpyridinium chloride extraction. Anal Biochem. 2004;329(1):77-84.

39. Nebuloni L, Kuhn GA, Müller R. A comparative analysis of water-soluble and blood-pool contrast agents for in vivo vascular imaging with micro-CT. Acad Radiol. 2013;20(10):1247-1255.

40. Chen Y, et al. Human extramedullary bone marrow in mice: a novel in vivo model of genetically controlled hematopoietic microenvironment. Blood. 2012;119(21):4971-4980.

41. Zaheer A, Lenkinski RE, Mahmood A, Jones AG, Cantley LC, Frangioni JV. In vivo near-infrared fluorescence imaging of osteoblastic activity. Nat Biotechnol. 2001;19(12):1148-1154.

42. Sonnylal S, et al. Connective tissue growth factor causes EMT-like cell fate changes in vivo and in vitro. J Cell Sci. 2013;126(Pt 10):2164-2175.

43. Morikawa S, et al. Prospective identification, isolation, and systemic transplantation of multipotent mesenchymal stem cells in murine bone marrow. J Exp Med. 2009;206(11):2483-2496.

44. Chartoff $\mathrm{EH}$, et al. Detection of intranasally delivered bone marrow-derived mesenchymal stromal cells in the lesioned mouse brain: a cautionary report. Stem Cells Int. 2011;2011:586586. 